This is a self-archived version of an original article. This version may differ from the original in pagination and typographic details.

Author(s): Harrach, Bastian; Pohjola, Valter; Salo, Mikko

Title: Dimension Bounds in Monotonicity Methods for the Helmholtz Equation

Year: 2019

Version: Published version

Copyright: (C) 2019 Society for Industrial and Applied Mathematics

Rights: In Copyright

Rights url: http://rightsstatements.org/page/lnC/1.0/?language=en

Please cite the original version:

Harrach, B., Pohjola, V., \& Salo, M. (2019). Dimension Bounds in Monotonicity Methods for the Helmholtz Equation. SIAM Journal on Mathematical Analysis, 51(4), 2995-3019.

https://doi.org/10.1137/19M1240708 


\title{
DIMENSION BOUNDS IN MONOTONICITY METHODS FOR THE HELMHOLTZ EQUATION*
}

\author{
BASTIAN HARRACH ${ }^{\dagger}$, VALTER POHJOLA ${ }^{\ddagger}$, AND MIKKO SALO ${ }^{\S}$
}

\begin{abstract}
The article [B. Harrach, V. Pohjola, and M. Salo, Anal. PDE] established a monotonicity inequality for the Helmholtz equation and presented applications to shape detection and local uniqueness in inverse boundary problems. The monotonicity inequality states that if two scattering coefficients satisfy $q_{1} \leq q_{2}$, then the corresponding Neumann-to-Dirichlet operators satisfy $\Lambda\left(q_{1}\right) \leq \Lambda\left(q_{2}\right)$ up to a finite-dimensional subspace. Here we improve the bounds for the dimension of this space. In particular, if $q_{1}$ and $q_{2}$ have the same number of positive Neumann eigenvalues, then the finite-dimensional space is trivial.
\end{abstract}

Key words. inverse problems, Helmholtz equation, montonicity method

AMS subject classification. $35 \mathrm{R} 30$

DOI. $10.1137 / 19 \mathrm{M} 1240708$

1. Introduction. This article is concerned with monotonicity properties arising in inverse problems and applications. As a basic example, if $\sigma_{1}$ and $\sigma_{2}$ are positive functions (representing electrical conductivities) in a bounded domain $\Omega \subset \mathbb{R}^{n}$ and if $\Lambda\left(\sigma_{1}\right)$ and $\Lambda\left(\sigma_{2}\right)$ are the corresponding Neumann-to-Dirichlet (ND) operators (representing electrical boundary measurements), then one has the monotonicity property

$$
\sigma_{1} \leq \sigma_{2} \Longrightarrow \Lambda\left(\sigma_{1}\right) \geq \Lambda\left(\sigma_{2}\right)
$$

The last statement means that $\Lambda\left(\sigma_{1}\right)-\Lambda\left(\sigma_{2}\right)$ is a positive semidefinite operator on the mean-free functions in $L^{2}(\partial \Omega)$ (the so-called Loewner order). This property, together with a certain nontrivial converse based on localized potentials [9], leads to efficient monotonicity based methods for determining shapes of obstacles or inclusions from electrical or optical boundary measurements (cf. [37] for the origin of this idea, [26] for the proof of the converse monotonicity property, and the list of references for recent works on monotonicity-based methods at the end of this introduction).

The recent work [24] extends monotonicity based methods to imaging problems with positive frequency, in particular acoustic imaging modeled by the Helmholtz equation. It turns out that the basic monotonicity property may fail in this case, but monotonicity still holds up to a finite-dimensional space and [24] shows that shape detection methods and local uniqueness results can be developed also in this situation. The paper [12] extends this idea to farfield inverse scattering and shows numerical reconstructions.

\footnotetext{
* Received by the editors January 25, 2019; accepted for publication (in revised form) April 17, 2019; published electronically July 25, 2019.

https://doi.org/10.1137/19M1240708

Funding: The work of the third author was supported by the Academy of Finland (Centre of Excellence in Inverse modeling and Imaging) grants 312121, 309963, and by the European Research Council under Horizon 2020 grant ERC CoG 770924.

†Institute for Mathematics, Goethe-University Frankfurt, Frankfurt am Main, Germany (harrach@math.uni-frankfurt.de).

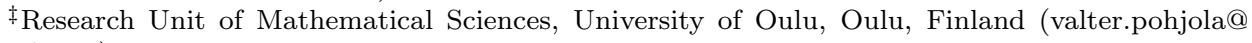
gmail.com).

$\S$ Department of Mathematics and Statistics, University of Jyväskylä, Jyväskylä, Finland (mikko.j. salo@jyu.fi).
} 
Let us describe the results of [24] in more detail. Let $\Omega \subset \mathbb{R}^{n}, n \geq 2$, be a bounded Lipschitz domain, and let $q \in L^{\infty}(\Omega)$ be a real valued function with $q \not \equiv 0$. Let $k>0$, and consider the Neumann problem

$$
\left\{\begin{aligned}
\left(\Delta+k^{2} q\right) u & =0 \text { in } \Omega, \\
\partial_{\nu} u & =g \text { on } \partial \Omega .
\end{aligned}\right.
$$

We assume that $k>0$ is not a resonance frequency, which means that the Neumann problem has a unique solution $u \in H^{1}(\Omega)$ for any $g \in L^{2}(\partial \Omega)$. Define the ND operator

$$
\Lambda(q): L^{2}(\partial \Omega) \rightarrow L^{2}(\partial \Omega),\left.\quad g \mapsto u\right|_{\partial \Omega} .
$$

Let $\lambda_{1} \geq \lambda_{2} \geq \cdots \rightarrow-\infty$ be the Neumann eigenvalues of $\Delta+k^{2} q$ in $\Omega$, and let $d(q)$ be the number of positive Neumann eigenvalues (counted with multiplicity).

In $[24$, Theorem 3.5] it was proved that

$$
q_{2}-q_{1} \geq 0 \Longrightarrow \Lambda\left(q_{2}\right)-\Lambda\left(q_{1}\right) \text { has only finitely many negative eigenvalues. }
$$

Here we consider $\Lambda\left(q_{2}\right)-\Lambda\left(q_{1}\right)$ as a compact self-adjoint operator on $L^{2}(\partial \Omega)$. Let $d\left(q_{1}, q_{2}\right)$ be the number of negative eigenvalues of $\Lambda_{q_{2}}-\Lambda_{q_{1}}$ (counting with multiplicity). In [24, Theorem 3.5] it was also proved that $d\left(q_{1}, q_{2}\right)$ satisfies the bound

$$
d\left(q_{1}, q_{2}\right) \leq d\left(q_{2}\right) .
$$

The next result gives a more precise estimate for $d\left(q_{1}, q_{2}\right)$.

THEOREM 1.1. Let $q_{1}, q_{2} \in L^{\infty}(\Omega) \backslash\{0\}$ be such that $k$ is not a resonance frequency for $q_{1}$ and $q_{2}$. Assume that $q_{1} \leq q_{2}$ a.e. in $\Omega$. Then

$$
d\left(q_{1}, q_{2}\right) \leq d\left(q_{2}\right)-d\left(q_{1}\right) .
$$

This has an immediate consequence: even if $q_{1}$ and $q_{2}$ are positive, the standard monotonicity inequality for the ND operators remains true if $q_{1}$ and $q_{2}$ have the same number of positive Neumann eigenvalues.

TheOREM 1.2. Let $q_{1}, q_{2} \in L^{\infty}(\Omega) \backslash\{0\}$ be such that $k$ is not a resonance frequency for $q_{1}$ and $q_{2}$. Assume that $d\left(q_{1}\right)=d\left(q_{2}\right)$. Then

$$
q_{1} \leq q_{2} \Longrightarrow \Lambda\left(q_{1}\right) \leq \Lambda\left(q_{2}\right) .
$$

Let us describe the main idea of the proof of Theorem 1.1. If $q_{1}$ and $q_{2}$ are in $L^{\infty}(\Omega) \backslash\{0\}$ and satisfy $q_{1} \leq q_{2}$, we define the interpolated potentials

$$
q(t)=q_{1}+t\left(q_{2}-q_{1}\right), \quad t \in[0,1] .
$$

Denote by $\lambda_{1}(t) \geq \lambda_{2}(t) \geq \cdots \rightarrow-\infty$ the Neumann eigenvalues of $\Delta+k^{2} q(t)$ in $\Omega$. Assume for simplicity that each $\lambda_{j}(t)$ is a simple eigenvalue. (The proof in section 2 removes this restriction.) Then each map $\lambda_{j}:[0,1] \rightarrow \mathbb{R}$ is smooth and strictly increasing. This follows from the variational formula

$$
\lambda_{j}^{\prime}(t)=k^{2} \int_{\Omega}\left(q_{2}-q_{1}\right) \varphi_{j}(t)^{2} d x
$$

where $\left\{\varphi_{j}(t)\right\}$ is an $L^{2}$-orthonormal basis consisting of Neumann eigenfunctions corresponding to $\left\{\lambda_{j}(t)\right\}$, and from the unique continuation principle. Now, when $t=0$ one starts with $d\left(q_{1}\right)$ positive eigenvalues, and when $t=1$ one has $d\left(q_{2}\right)$ positive 
eigenvalues. Since the maps $t \mapsto \lambda_{j}(t)$ are strictly increasing, exactly $d\left(q_{2}\right)-d\left(q_{1}\right)$ eigenvalues cross the real axis as $t$ increases to 1 , and the eigenspace at each crossing gives rise to a one-dimensional subspace of $L^{2}(\partial \Omega)$. Now if $g \in L^{2}(\partial \Omega)$ is orthogonal to all these one-dimensional subspaces, it follows that $\left(\left(\Lambda\left(q_{2}\right)-\Lambda\left(q_{1}\right)\right) g, g\right) \geq 0$, proving that the finite-dimensional obstruction has dimension $\leq d\left(q_{2}\right)-d\left(q_{1}\right)$.

The next result complements Theorem 1.1 by showing that in certain cases where $q_{1}$ and $q_{2}$ differ by a constant, there are lower bounds on the number of negative eigenvalues. Its proof is based on computing an expression for the quadratic form $\left(\left(\Lambda\left(q_{2}-b\right)-\Lambda\left(q_{1}+a\right)\right) g, g\right)$ in terms of the Neumann eigenfunctions of $\Delta+k^{2} q_{1}$ and showing that the quadratic form is negative for $g$ in a space spanned by finitely many traces of Neumann eigenfunctions.

THEOREM 1.3. Let $q_{1}, q_{2} \in L^{\infty}(\Omega) \backslash\{0\}$ be such that $k$ is nonresonant for $q_{1}$ and $q_{2}$. Assume that $q_{2}-q_{1}$ is a positive constant and $d\left(q_{2}\right)>d\left(q_{1}\right)$. Let $\mu_{1}$ be the largest negative Neumann eigenvalue of $\Delta+k^{2} q_{1}$, and let $\mu_{2}$ be the smallest positive eigenvalue of $\Delta+k^{2} q_{2}$. Let $N_{j}$ be the multiplicity of $\mu_{j}$.

(a) $\Lambda\left(q_{2}-b\right)-\Lambda\left(q_{1}+a\right)$ has at least $N_{1}$ negative eigenvalues whenever $a<k^{-2}\left|\mu_{1}\right|$ is sufficiently close to $k^{-2}\left|\mu_{1}\right|$, and $b \in\left[0, k^{-2} \mu_{2}\right)$.

(b) $\Lambda\left(q_{2}-b\right)-\Lambda\left(q_{1}+a\right)$ has at least $N_{2}$ negative eigenvalues whenever $b<k^{-2} \mu_{2}$ is sufficiently close to $k^{-2} \mu_{2}$, and $a \in\left[0, k^{-2}\left|\mu_{1}\right|\right)$.

From the previous theorem, we obtain the following special cases where equality is attained in Theorem 1.1.

THEOREM 1.4.

(a) Let $q \in L^{\infty}(\Omega)$ be such that 0 is a Neumann eigenvalue of $\Delta+k^{2} q$ with multiplicity $N$. For $\varepsilon>0$ small enough, $d(q+\varepsilon)-d(q-\varepsilon)=N$ and $\Lambda(q+$ $\varepsilon)-\Lambda(q-\varepsilon)$ has exactly $N$ negative eigenvalues.

(b) Let $\Omega$ be the square $(0, \pi)^{2} \subset \mathbb{R}^{2}$, let $k>0$, and let $N \geq 2$ be even. There is a $c>0$ such that for $\varepsilon>0$ small, $\Lambda(c+\varepsilon)-\Lambda(c-\varepsilon)$ has exactly $N=$ $d(c+\varepsilon)-d(c-\varepsilon)$ negative eigenvalues.

Let us give some more references to earlier and related work and comment on the relevance of our results. Monotonicity estimates and localized potentials techniques have been used in different ways for the study of inverse problems $[14,25,15,1$, $26,2,28,4,12,24,18,20]$ and several recent works build practical reconstruction methods on monotonicity properties [37, 17, 27, 22, 30, 38, 6, 7, 36, 39, 23, 40, 8]. Recently, monotonicity arguments were also discovered to yield Lipschitz stability results (cf. $[21,35,16])$. All of these works consider stationary imaging cases where monotonicity of the ND operators holds in the sense of the Loewner order as explained above. So far, only $[24,12,19]$ cover the case of positive frequency imaging where the monotonicity only holds up to a finite-dimensional space. For extending monotonicitybased theoretical uniqueness and stability results, as well as monotonicity-based numerical reconstruction methods, it seems to be of utmost importance to have a good bound on the number of eigenvalues that have to be disregarded. [24] showed that this number is smaller than $d\left(q_{2}\right)$, which might become arbitrarily large for high frequencies $k \rightarrow \infty$. Using this bound would result in disregarding a large part of the ND operators for high frequencies and might make numerical reconstruction methods unfeasible. This article, however, shows that the number is smaller than $d\left(q_{2}\right)-d\left(q_{1}\right)$ which might still be small (or even zero) for high frequencies. Note also that this article indicates that the bound is sharp for $q_{1}$ close to $q_{2}$, but that the bound might get too large when $q_{2}-q_{1}$ increases (cf. section 4 ). 
The rest of this paper is organized as follows. Section 2 gives the proof of Theorem 1.1, and section 3 proves Theorems 1.3 and 1.4. Section 4 gives a simple alternative proof of Theorem 1.1 for the case where $q_{1}$ and $q_{2}$ are constant and numerically studies the sharpness of the bound for large $q_{2}-q_{1}$.

2. Upper bound for the number of negative eigenvalues. For the proof of Theorem 1.1, it will be useful to consider solutions of the Helmholtz equation also when $k$ is a resonant frequency. In this case the Neumann data needs to satisfy finitely many linear constraints.

Lemma 2.1. Let $q \in L^{\infty}(\Omega)$ and $k>0$, and define the sets

$$
\begin{aligned}
& N(q)=\left\{\varphi \in H^{1}(\Omega) ;\left(\Delta+k^{2} q\right) \varphi=0 \text { in } \Omega,\left.\partial_{\nu} \varphi\right|_{\partial \Omega}=0\right\}, \\
& D(q)=\left\{\left.\varphi\right|_{\partial \Omega} ; \varphi \in N(q)\right\} .
\end{aligned}
$$

Also let $N(q)^{\perp}$ be the orthogonal complement of $N(q)$ in $L^{2}(\Omega)$, and let $D(q)^{\perp}$ be the orthogonal complement of $D(q)$ in $L^{2}(\partial \Omega)$.

(a) $N(q)$ and $D(q)$ are finite-dimensional spaces whose dimension is the multiplicity of 0 as the Neumann eigenvalue of $\Delta+k^{2} q$ in $\Omega$.

(b) For any $F \in L^{2}(\Omega)$ and $g \in L^{2}(\partial \Omega)$, the equation

$$
\left(\Delta+k^{2} q\right) u=F \text { in } \Omega,\left.\quad \partial_{\nu} u\right|_{\partial \Omega}=g,
$$

has a solution $u \in H^{1}(\Omega)$ if and only if one has the compatibility conditions

$$
\int_{\Omega} F \varphi d x=\int_{\partial \Omega} g \varphi d S, \quad \varphi \in N(q) .
$$

In particular, a solution exists whenever $F \in N(q)^{\perp}$ and $g \in D(q)^{\perp}$. The solution is unique up to addition of a function in $N(q)$, and one has a bounded map

$$
T_{q}: N(q)^{\perp} \times D(q)^{\perp} \rightarrow H^{1}(\Omega), \quad(F, g) \mapsto u_{F, g},
$$

where $u_{F, g}$ is the unique solution with $(u, \varphi)_{H^{1}(\Omega)}=0$ for $\varphi \in N(q)$.

(c) Let $\lambda_{1} \geq \lambda_{2} \geq \lambda_{3} \geq \cdots \rightarrow-\infty$ be the Neumann eigenvalues of $\Delta+k^{2} q$ in $\Omega$ and let $\left(\varphi_{j}\right)_{j=1}^{\infty}$ be a corresponding orthonormal basis of $L^{2}(\Omega)$ consisting of Neumann eigenfunctions. If $F \in L^{2}(\Omega)$ and $g \in L^{2}(\partial \Omega)$ satisfy (2.2), then any solution $u \in H^{1}(\Omega)$ of $(2.1)$ may be represented as the $L^{2}(\Omega)$-convergent sum

$$
u=\sum_{j \in J} a_{j} \varphi_{j}+\sum_{j \notin J} \frac{1}{\lambda_{j}}\left[\int_{\Omega} F \varphi_{j} d x-\int_{\partial \Omega} g \varphi_{j} d S\right] \varphi_{j},
$$

where $J=\left\{j \geq 1 ; \lambda_{j}=0\right\}$ is finite, and $a_{j} \in \mathbb{R}$ are some constants.

Remark. The sum in part (c) may not converge in higher norms in general. In fact, if it did converge in some space where the normal derivative operator is bounded, then one would get that $\left.\partial_{\nu} u\right|_{\partial \Omega}=0$, which is not true if $g \neq 0$.

Proof. As in [24, section 2.1] we use the compact inclusion map $\iota: H^{1}(\Omega) \rightarrow$ $L^{2}(\Omega)$ to define $K=\iota^{*} \iota$ and $K_{q}=\iota^{*} M_{q} \iota$, where $M_{q}: L^{2}(\Omega) \rightarrow L^{2}(\Omega)$ is the multiplication operator by $q$. Both $K$ and $K_{q}$ are compact self-adjoint operators from $H^{1}(\Omega)$ to $H^{1}(\Omega)$. A function $u \in H^{1}(\Omega)$ is a weak solution of (2.1) if and only if

$$
\left(\mathrm{Id}-K-k^{2} K_{q}\right) u=-\iota^{*} F+\gamma^{*} g,
$$


where $\gamma: H^{1}(\Omega) \rightarrow L^{2}(\partial \Omega)$ is the trace operator. By Fredholm theory (see, e.g., [34, Corollary 8.95]), this problem has a solution $u \in H^{1}(\Omega)$ for given $F \in L^{2}(\Omega)$, $g \in L^{2}(\partial \Omega)$ if and only if

$$
\left(-\iota^{*} F+\gamma^{*} g, \varphi\right)_{H^{1}(\Omega)}=0
$$

for all $\varphi \in H^{1}(\Omega)$ in the kernel of Id $-K-k^{2} K_{q}$. But this finite-dimensional kernel is equal to $N(q)$, showing that (2.1) is solvable if and only if (2.2) holds. The representation in [34, Corollary 8.95] shows that there is a unique solution $u_{F, g}$ with $\left(u_{F, g}, \varphi\right)_{H^{1}(\Omega)}=0$ for $\varphi \in N(q)$ and that

$$
\left\|u_{F, g}\right\|_{H^{1}(\Omega)} \leq C\left\|-\iota^{*} F+\gamma^{*} g\right\|_{H^{1}(\Omega)} \leq C\left(\|F\|_{L^{2}(\Omega)}+\|g\|_{L^{2}(\partial \Omega)}\right) .
$$

Finally, the map $N(q) \rightarrow D(q),\left.\varphi \mapsto \varphi\right|_{\partial \Omega}$ is bijective by the unique continuation principle. This proves (a) and (b).

To prove (c) let $u$ be a solution of (2.1). Since $\left(\varphi_{j}\right)$ is an orthonormal basis of $L^{2}(\Omega)$ we have

$$
u=\sum_{j=1}^{\infty} c_{j} \varphi_{j}, \quad c_{j}=\int_{\Omega} u \varphi_{j} d x
$$

with convergence in $L^{2}(\Omega)$. Testing the weak form of $(2.1)$ against $\varphi_{j}$ and integrating by parts gives that

$$
\begin{aligned}
\int_{\Omega} F \varphi_{j} d x & =\left(\left(\Delta+k^{2} q\right) u, \varphi_{j}\right)_{L^{2}(\Omega)}=\int_{\partial \Omega} g \varphi_{j} d S+\left(u,\left(\Delta+k^{2} q\right) \varphi_{j}\right)_{L^{2}(\Omega)} \\
& =\int_{\partial \Omega} g \varphi_{j} d S+\lambda_{j} \int_{\Omega} u \varphi_{j} .
\end{aligned}
$$

This yields the representation for $u$ in (c).

For $q_{1}, q_{2} \in L^{\infty}(\Omega) \backslash\{0\}$, we define

$$
q(t):=q_{1}+t\left(q_{2}-q_{1}\right), \quad t \in[0,1] .
$$

We also define the family of operators

$$
H(t):=\Delta+k^{2} q(t), \quad t \in[0,1] .
$$

The following result from analytic perturbation theory is needed to describe the behavior of the eigenvalues of $H(t)$ as $t$ changes.

LEMmA 2.2. Let $q_{1}, q_{2} \in L^{\infty}(\Omega)$ and $k>0$, assume that $q_{1} \leq q_{2}$ and $q_{1} \not \equiv q_{2}$, and let $\lambda_{1} \geq \lambda_{2} \geq \cdots \rightarrow-\infty$ be the Neumann eigenvalues of $\Delta+k^{2} q_{1}$ in $\Omega$. There exist real-analytic functions $\lambda_{j}:[0,1] \rightarrow \mathbb{R}$ and $\varphi_{j}:[0,1] \rightarrow L^{2}(\Omega)$ with the following properties:

(a) $\lambda_{j}(0)=\lambda_{j}$ for $j \geq 1$, and for any $t \in[0,1]$, the numbers $\lambda_{j}(t)$ represent the repeated ${ }^{1}$ Neumann eigenvalues of $H(t)$ in $\Omega$. Zero is a Neumann eigenvalue of $H\left(t_{0}\right)$ with multiplicity $N$ if and only if precisely $N$ functions $\lambda_{j}(t)$ vanish at $t=t_{0}$.

(b) Each $\lambda_{j}(t)$ is strictly increasing on $[0,1]$.

\footnotetext{
${ }^{1}$ We call $\lambda_{1} \geq \lambda_{2} \geq \ldots$ the repeated eigenvalues if and only if the value $\lambda_{j}$ is repeated the number of times of its multiplicity in the sequence.
} 
(c) Each $\varphi_{j}(t)$ is a Neumann eigenfunction in $H^{1}(\Omega)$ satisfying

$$
H(t) \varphi_{j}(t)=\lambda_{j}(t) \varphi_{j}(t) \text { in } \Omega,\left.\quad \partial_{\nu} \varphi_{j}(t)\right|_{\partial \Omega}=0,
$$

and $\left(\varphi_{j}(t)\right)_{j=1}^{\infty}$ is an orthonormal basis of $L^{2}(\Omega)$ for any $t \in[0,1]$. Each map $[0,1] \rightarrow H^{1}(\Omega), t \mapsto \varphi_{j}(t)$ is real-analytic.

Proof. This result will be proved by analytic perturbation theory, and hence in this proof we will assume the function spaces to be complex valued.

(a) For $z \in \mathbb{C}$, define the operator

$$
H(z)=\Delta+k^{2}\left(q_{1}+z\left(q_{2}-q_{1}\right)\right) .
$$

Then $H(t)=\Delta+k^{2} q(t)$ for $t \in \mathbb{R}$. We wish to use [29, Theorem VII.3.9, p. 392] to show that the Neumann eigenvalues of $H(t)$ can be parametrized analytically with respect to $t$ (see [3, Theorem 3.1, p. 442] and [32, Theorem XII.13] for related results). In order to do this, we need to realize $H(z)$ with Neumann boundary values as a selfadjoint analytic family of unbounded operators on $L^{2}(\Omega)$. In the present case where $\Omega$ has Lipschitz boundary, the required results may be found in [10, section 2]. (In fact the easier abstract results in [10, Appendix B] would suffice.)

Define the set

$$
\mathcal{D}=\left\{u \in H^{1}(\Omega) ; \Delta u \in L^{2}(\Omega), \tilde{\gamma}_{N} u=0 \text { in } H^{-1 / 2}(\partial \Omega)\right\},
$$

where $\tilde{\gamma}_{N}$ is the weak Neumann trace operator in [10, Formulas (2.40) and (2.41)]. We consider $H(z)$ as an unbounded linear operator on $L^{2}(\Omega)$ with domain $\operatorname{dom}(H(z))=$ $\mathcal{D}$. The family $(H(z))_{z \in \mathbb{C}}$ has the following properties:

(i) Each $H(z)$ is closed and densely defined. This follows since $\Delta$ with domain $\mathcal{D}$ is self-adjoint by [10, Theorem 2.6], and hence $\Delta$ and consequently also $H(z)$ is closed and densely defined.

(ii) The family $(H(z))_{z \in \mathbb{C}}$ is holomorphic of type (A) (see [29, section VII.2.1]) since for each $u \in \mathcal{D}$, the map

$$
z \mapsto H(z) u=\Delta u+k^{2} q_{1} u+k^{2} z\left(q_{2}-q_{1}\right) u
$$

is holomorphic.

(iii) The family $(H(z))_{z \in \mathbb{C}}$ is a self-adjoint holomorphic family, i.e., a holomorphic family of operators satisfying $H(z)^{*}=H(\bar{z})$ (see [29, section VII.3.1]): since $\Delta$ with domain $\mathcal{D}$ is self-adjoint [10, Theorem 2.6] and the map $B(z): u \mapsto$ $k^{2}\left(q_{1}+z\left(q_{2}-q_{1}\right)\right) u$ is bounded on $L^{2}(\Omega)$, by [5, Lemma XII.1.6] we have

$$
H(z)^{*}=\Delta^{*}+B(z)^{*}=\Delta+B(\bar{z})=H(\bar{z}) .
$$

(iv) $H(z)$ has a compact resolvent, when $z \in \mathbb{C}$. This can be seen as follows. Let

$$
R_{z}(\zeta):=(H(z)-\zeta)^{-1},
$$

denote the resolvent. Arguing as in the proof of [10, Corollary 2.7] using [10, Remark 2.19], we have that

$$
R_{z}\left(\zeta_{0}\right):=\left(H(z)-\zeta_{0}\right)^{-1}
$$

is compact, when $\zeta_{0} \in \mathbb{R}_{+}$is large enough. By the resolvent identity in [31, Theorem VIII.2] we have that

$$
R_{z}(\zeta)=R_{z}\left(\zeta_{0}\right)-\left(\zeta_{0}-\zeta\right) R_{z}(\zeta) R_{z}\left(\zeta_{0}\right)
$$


The resolvent $R_{z}(\zeta)$ is by definition continuous on $L^{2}(\Omega)$, when $\zeta$ is in the resolvent set. The above formula hence implies that $R_{z}(\zeta)$ is compact on $L^{2}(\Omega)$, since $R_{z}\left(\zeta_{0}\right)$ is compact.

Thus the family $(H(z))_{z \in \mathbb{C}}$ satisfies the conditions in [29, Theorem VII.3.9, p. 392], and there are real-analytic functions $\lambda_{j}(t)$ and real-analytic vector functions $\varphi_{j}(t)$, for $t \in[0,1]$, such that $\lambda_{j}(t)$ represent all the repeated eigenvalues of $H(t), \varphi_{j}(t)$ are the corresponding eigenfunctions, and $\left(\varphi_{j}(t)\right)$ is an orthonormal basis of $L^{2}(\Omega)$. Since $\varphi_{j}(t) \in \mathcal{D}$, these are exactly the standard Neumann eigenvalues and eigenfunctions of $H(t)$ (see $[10$, Formula $(2.41)]$ ). We may reorder $\lambda_{j}(t)$ and $\varphi_{j}(t)$ so that $\lambda_{j}(0)=\lambda_{j}$.

(b) We compute $\lambda_{j}^{\prime}(t)$ using a variational formula: by [29, Formula (VII.3.18), p. $391]$ and by the fact that $H^{\prime}(t) u=k^{2}\left(q_{2}-q_{1}\right) u$, we have

$$
\lambda_{j}^{\prime}(t)=\left(H^{\prime}(t) \varphi_{j}(t), \varphi_{j}(t)\right)_{L^{2}(\Omega)}=\int_{\Omega} k^{2}\left(q_{2}-q_{1}\right) \varphi_{j}(t)^{2} d x .
$$

Using the assumption that $q_{2} \geq q_{1}$, we have $\lambda_{j}^{\prime}(t) \geq 0$. Moreover, since $q_{1} \not \equiv q_{2}$, we have $q_{2}-q_{1} \geq c>0$ in some set $E$ of positive measure in $\Omega$. Thus we see that $\lambda_{j}^{\prime}(t)>0$. (Otherwise if $\lambda_{j}^{\prime}(t)=0$, then $\int_{E} \varphi_{j}(t)^{2} d x=0$ which would contradict the unique continuation principle.) This implies that each $\lambda_{j}(t)$ is a strictly increasing function on $[0,1]$.

(c) All other statements in (c) have been proved, except that $t \mapsto \varphi_{j}(t)$ is realanalytic as an $H^{1}(\Omega)$-valued function. To prove this, note first that for any $v \in L^{2}(\Omega)$ the map

$$
t \mapsto\left(\varphi_{j}(t), v\right)_{L^{2}(\Omega)}
$$

is real-analytic on $[0,1]$. Now, if $\psi \in H^{1}(\Omega)$, we compute

$$
\begin{aligned}
\left(\varphi_{j}(t), \psi\right)_{H^{1}(\Omega)} & =\left(\varphi_{j}(t), \psi\right)_{L^{2}(\Omega)}-\left(\Delta \varphi_{j}(t), \psi\right)_{L^{2}(\Omega)} \\
& =\left(\varphi_{j}(t), \psi\right)_{L^{2}(\Omega)}+k^{2}\left(q(t) \varphi_{j}(t), \psi\right)_{L^{2}(\Omega)}-\lambda_{j}(t)\left(\varphi_{j}(t), \psi\right)_{L^{2}(\Omega)}
\end{aligned}
$$

Each term on the last line is real-analytic for $t \in[0,1]$. Thus $t \mapsto \varphi_{j}(t)$ is weakly, and hence strongly, analytic as an $H^{1}(\Omega)$-valued function.

We will next combine Lemmas 2.1 and 2.2 to obtain solutions of

$$
\left(\Delta+k^{2} q(t)\right) u_{t}=0 \text { in } \Omega,\left.\quad \partial_{\nu} u_{t}\right|_{\partial \Omega}=g,
$$

that depend Lipschitz continuously on $t \in[0,1]$ as long as $g$ is orthogonal to a finitedimensional subspace of $L^{2}(\partial \Omega)$.

LEMMA 2.3. Assume the conditions in Lemma 2.2. Let $t_{1}<\cdots<t_{K}$ be the times when 0 is a Neumann eigenvalue of $H(t)$, let $g \in L^{2}(\partial \Omega)$, and let $u_{t}$ be the unique solution of (2.4) for $t \in[0,1] \backslash\left\{t_{1}, \ldots, t_{K}\right\}$.

(a) The map

$$
[0,1] \backslash\left\{t_{1}, \ldots, t_{K}\right\} \rightarrow H^{1}(\Omega), \quad t \mapsto u_{t},
$$

is real-analytic. The derivative $\partial_{t} u_{t}$ is the unique $H^{1}(\Omega)$ solution of $(\Delta+$ $\left.k^{2} q(t)\right) v=-k^{2} q^{\prime}(t) u_{t}$ in $\Omega$ with $\left.\partial_{\nu} v\right|_{\partial \Omega}=0$. For any compact $F \subset[0,1] \backslash$ $\left\{t_{1}, \ldots, t_{K}\right\}$, there is $C_{F}>0$ such that

$$
\left\|u_{t}\right\|_{H^{1}(\Omega)} \leq C_{F}\|g\|_{L^{2}(\partial \Omega)}, \quad t \in F
$$


(b) Let $t_{0}$ be one of $t_{1}, \ldots, t_{K}$ and let $I=\left\{j \geq 1 ; \lambda_{j}\left(t_{0}\right)=0\right\}$. Then

$$
\left\|u_{t}-\sum_{j \in I}\left(u_{t}, \varphi_{j}(t)\right)_{L^{2}(\Omega)} \varphi_{j}(t)\right\|_{H^{1}(\Omega)} \leq C\|g\|_{L^{2}(\partial \Omega)}
$$

uniformly for $t$ close to $t_{0}$.

(c) If $J=\left\{j \geq 1 ; \lambda_{j}\left(t_{l}\right)=0\right.$ for some $\left.l \in\{1,2, \ldots, K\}\right\}$, then

$$
\left\|u_{t}-\sum_{j \in J}\left(u_{t}, \varphi_{j}(t)\right)_{L^{2}(\Omega)} \varphi_{j}(t)\right\|_{H^{1}(\Omega)} \leq C\|g\|_{L^{2}(\partial \Omega)}
$$

uniformly over $t \in[0,1] \backslash\left\{t_{1}, \ldots, t_{K}\right\}$.

(d) With the notation of Lemma 2.1 let

$$
D=D\left(q\left(t_{1}\right)\right) \oplus \cdots \oplus D\left(q\left(t_{K}\right)\right) .
$$

If additionally $g \in D^{\perp}$, then $t \mapsto u_{t}$ extends uniquely as a Lipschitz continuous map

$$
[0,1] \rightarrow H^{1}(\Omega), \quad t \mapsto u_{t} .
$$

Proof. We first show that 0 is a Neumann eigenvalue of $H(t)$ at only finitely many times $t$. Note that $\Delta+k^{2} q_{2}$ has at most finitely many positive Neumann eigenvalues. Since the functions $\lambda_{j}(t)$ are strictly increasing and since the Neumann eigenvalues of $\Delta+k^{2} q_{2}$ are given by $\lambda_{j}(1)$, we see that only finitely many of the functions $\lambda_{j}(t)$ have a zero in $[0,1]$. Thus there are only finitely many times $0 \leq t_{1}<\cdots<t_{K} \leq 1$ in the interval $t \in[0,1]$ so that 0 is a Neumann eigenvalue of $H(t)$.

Proof of part (a). Fix any $g \in L^{2}(\partial \Omega)$ and let $t \in[0,1] \backslash\left\{t_{1}, \ldots, t_{K}\right\}$. Then the Neumann problem for $\Delta+k^{2} q(t)$ in $\Omega$ is well-posed, and we define $u_{t}=T_{q(t)}(0, g)$ as the unique solution of (2.4). Fix $t_{0} \in[0,1] \backslash\left\{t_{1}, \ldots, t_{K}\right\}$, and note that for $t$ close to $t_{0}$ one has

$$
\left(\Delta+k^{2} q\left(t_{0}\right)\right)\left(u_{t}-u_{t_{0}}\right)=-k^{2}\left(q(t)-q\left(t_{0}\right)\right) u_{t}=-k^{2}\left(t-t_{0}\right)\left(q_{2}-q_{1}\right) u_{t}
$$

and $\left.\partial_{\nu}\left(u_{t}-u_{t_{0}}\right)\right|_{\partial \Omega}=0$. It follows that

$$
\left[\operatorname{Id}+k^{2}\left(t-t_{0}\right) G\left(q\left(t_{0}\right)\right)\left(\left(q_{2}-q_{1}\right) \cdot\right)\right] u_{t}=u_{t_{0}},
$$

where $G\left(q\left(t_{0}\right)\right): F \mapsto T_{q\left(t_{0}\right)}(F, 0)$ is bounded $L^{2}(\Omega) \rightarrow H^{1}(\Omega)$ by Lemma 2.1. Choosing $t$ close to $t_{0}$, we can solve the last equation by Neumann series so that

$$
u_{t}=\sum_{j=0}^{\infty}\left(-k^{2}\right)^{j}\left(t-t_{0}\right)^{j}\left[G\left(q\left(t_{0}\right)\right)\left(\left(q_{2}-q_{1}\right) \cdot\right)\right]^{j} u_{t_{0}} .
$$

Thus $t \mapsto u_{t}$ is real-analytic in $[0,1] \backslash\left\{t_{1}, \ldots, t_{K}\right\}$. Moreover, for any $t_{0} \in[0,1] \backslash$ $\left\{t_{1}, \ldots, t_{K}\right\}$ there is $\varepsilon\left(t_{0}\right)>0$ so that

$$
\left\|u_{t}\right\|_{H^{1}(\Omega)} \leq 2\left\|u_{t_{0}}\right\|_{H^{1}(\Omega)} \leq C\left(t_{0}\right)\|g\|_{L^{2}(\partial \Omega)}, \quad\left|t-t_{0}\right|<\varepsilon\left(t_{0}\right) .
$$

This proves the uniform bound for $\left\|u_{t}\right\|_{H^{1}(\Omega)}$ over any compact subset $F$ of $[0,1] \backslash$ $\left\{t_{1}, \ldots, t_{K}\right\}$. Finally, differentiating the power series and evaluating at $t_{0}$ yields

$$
\left.\partial_{t} u_{t}\right|_{t=t_{0}}=-k^{2} G\left(q\left(t_{0}\right)\right)\left(\left(q_{2}-q_{1}\right) u_{t_{0}}\right)
$$


so that $\left.\partial_{t} u_{t}\right|_{t=t_{0}}$ is the unique solution of $\left(\Delta+k^{2} q\left(t_{0}\right)\right) v=-k^{2}\left(q_{2}-q_{1}\right) u_{t_{0}}$ in $\Omega$ with $\left.\partial_{\nu} v\right|_{\partial \Omega}=0$.

Proof of part (b). Let $t_{0}$ be one of $t_{1}, \ldots, t_{K}$. Let $I=\left\{j \geq 1 ; \lambda_{j}\left(t_{0}\right)=0\right\}$ (so that $I$ is finite), and write

$$
u_{t}=v_{t}+w_{t}, \quad v_{t}=P_{t} u_{t}, \quad w_{t}=Q_{t} u_{t},
$$

where $P_{t}$ and $Q_{t}$ are the orthogonal projections on $L^{2}(\Omega)$ given by

$$
P_{t} u=\sum_{j \in I}\left(u, \varphi_{j}(t)\right)_{L^{2}(\Omega)} \varphi_{j}(t), \quad Q_{t} u=\sum_{j \notin I}\left(u, \varphi_{j}(t)\right)_{L^{2}(\Omega)} \varphi_{j}(t) .
$$

We need to prove that $\left\|w_{t}\right\|_{H^{1}(\Omega)} \leq C\|g\|_{L^{2}(\partial \Omega)}$ for $t$ close to $t_{0}$.

Fix some $\bar{t} \in[0,1] \backslash\left\{t_{1}, \ldots, t_{K}\right\}$, and write $u_{t}=r+h_{t}$, where $r \in H^{1}(\Omega)$ is the unique solution of

$$
\left(\Delta+k^{2} q(\bar{t})\right) r=0 \text { in } \Omega,\left.\quad \partial_{\nu} r\right|_{\partial \Omega}=g .
$$

Then $h_{t}$ solves

$$
\left(\Delta+k^{2} q(t)\right) h_{t}=F_{t} \text { in } \Omega,\left.\quad \partial_{\nu} h_{t}\right|_{\partial \Omega}=0,
$$

where

$$
F_{t}=-\left(\Delta+k^{2} q(t)\right) r=-k^{2}(q(t)-q(\bar{t})) r=-k^{2}(t-\bar{t})\left(q_{2}-q_{1}\right) r .
$$

Now $w_{t}=Q_{t} u_{t}=Q_{t} r+Q_{t} h_{t}$, so that

$$
\left\|w_{t}\right\|_{L^{2}(\Omega)} \leq\|r\|_{L^{2}(\Omega)}+\left[\sum_{j \notin I}\left(\int_{\Omega} h_{t} \varphi_{j}(t) d x\right)^{2}\right]^{1 / 2} .
$$

Testing the equation for $h_{t}$ against $\varphi_{j}(t)$ and integrating by parts gives that

$$
\lambda_{j}(t) \int_{\Omega} h_{t} \varphi_{j}(t) d x=\int_{\Omega} F_{t} \varphi_{j}(t) d x
$$

and consequently

$$
\left\|w_{t}\right\|_{L^{2}(\Omega)} \leq\|r\|_{L^{2}(\Omega)}+\left[\sum_{j \notin I} \frac{1}{\lambda_{j}(t)^{2}}\left(\int_{\Omega} F_{t} \varphi_{j}(t) d x\right)^{2}\right]^{1 / 2} .
$$

Now, the main point is that $\left|\lambda_{j}\left(t_{0}\right)\right| \geq c>0$ for $j \notin I$. Moreover, the formula (2.3) implies that

$$
0 \leq \lambda_{j}^{\prime}(t) \leq C, \quad \text { uniformly over } j \geq 1 \text { and } t \in[0,1] .
$$

These facts imply that there is $\varepsilon>0$ so that

$$
\left|\lambda_{j}(t)\right| \geq c / 2, \quad \text { uniformly over } j \notin I \text { and }\left|t-t_{0}\right| \leq \varepsilon .
$$

It follows that

$$
\left\|w_{t}\right\|_{L^{2}(\Omega)} \leq\|r\|_{L^{2}(\Omega)}+\frac{2}{c}\left\|F_{t}\right\|_{L^{2}(\Omega)}, \quad\left|t-t_{0}\right| \leq \varepsilon .
$$


Thus $\left\|w_{t}\right\|_{L^{2}(\Omega)} \leq C\|g\|_{L^{2}(\partial \Omega)}$ uniformly over $t \in\left[t_{0}-\varepsilon, t_{0}+\varepsilon\right]$, since $\|r\|_{H^{1}(\Omega)} \leq$ $C\|g\|_{L^{2}(\partial \Omega)}$ and $F_{t}=-k^{2}(t-\bar{t})\left(q_{2}-q_{1}\right) r$.

Finally we estimate the $H^{1}$ norm. By Lemma 2.1, we have that

$$
v_{t}=-\sum_{j \in I} \frac{1}{\lambda_{j}(t)}\left[\int_{\partial \Omega} g \varphi_{j}(t) d S\right] \varphi_{j}(t)
$$

Now since $w_{t}$ solves the equation

$$
\left(\Delta+k^{2} q(t)\right) w_{t}=G_{t} \text { in } \Omega,\left.\quad \partial_{\nu} w_{t}\right|_{\partial \Omega}=g,
$$

where by (2.6)

$$
G_{t}=-\left(\Delta+k^{2} q(t)\right) v_{t}=-\sum_{j \in I}\left[\int_{\partial \Omega} g \varphi_{j}(t) d S\right] \varphi_{j}(t),
$$

we obtain that

$$
\begin{aligned}
\left\|\nabla w_{t}\right\|_{L^{2}(\Omega)}^{2} & =\int_{\Omega}\left(-\Delta w_{t}\right) w_{t} d x+\int_{\partial \Omega}\left(\partial_{\nu} w_{t}\right) w_{t} d S \\
& =\int_{\Omega}\left(k^{2} q(t) w_{t}-G_{t}\right) w_{t} d x+\int_{\partial \Omega} g w_{t} d S .
\end{aligned}
$$

Consequently

$$
\left\|w_{t}\right\|_{H^{1}(\Omega)}^{2} \leq C\left(\left\|w_{t}\right\|_{L^{2}(\Omega)}^{2}+\left\|G_{t}\right\|_{L^{2}(\Omega)}^{2}\right)+\int_{\partial \Omega} g w_{t} d S .
$$

Using Cauchy's inequality with $\varepsilon$ in the boundary integral and the trace result $\left\|w_{t}\right\|_{L^{2}(\partial \Omega)} \leq C\left\|w_{t}\right\|_{H^{1}(\Omega)}$, we obtain that

$$
\left\|w_{t}\right\|_{H^{1}(\Omega)}^{2} \leq C\left(\left\|w_{t}\right\|_{L^{2}(\Omega)}^{2}+\left\|G_{t}\right\|_{L^{2}(\Omega)}^{2}+\|g\|_{L^{2}(\partial \Omega)}^{2}\right) .
$$

We have seen above that $\left\|w_{t}\right\|_{L^{2}(\Omega)} \leq C\|g\|_{L^{2}(\partial \Omega)}$ uniformly over $\left|t-t_{0}\right| \leq \varepsilon$, and the same is true for $\left\|G_{t}\right\|_{L^{2}(\Omega)}$. Thus $\left\|w_{t}\right\|_{H^{1}(\Omega)} \leq C\|g\|_{L^{2}(\partial \Omega)}$ uniformly over $\left|t-t_{0}\right| \leq \varepsilon$.

Proof of part (c). This is completely analogous to the proof of part (b), upon using the fact that $\left|\lambda_{j}(t)\right| \geq c>0$ uniformly over $j \notin J$ and $t \in[0,1]$.

Proof of part (d). Let now $g \in D^{\perp}$, let $t_{0}=t_{l}$, where $1 \leq l \leq K$, and let $t \neq t_{0}$ be close to $t_{0}$. As in part (b), we write $u_{t}=v_{t}+w_{t}$, where $v_{t}=P_{t} u_{t}$ and $w_{t}=Q_{t} u_{t}$.

We first prove that under the assumption $g \in D^{\perp}$, the map $t \mapsto v_{t}$ is a realanalytic from $[0,1]$ to $H^{1}(\Omega)$. By $(2.6)$ we have that

$$
v_{t}=-\sum_{j \in I} \frac{1}{\lambda_{j}(t)}\left[\int_{\partial \Omega} g \varphi_{j}(t) d S\right] \varphi_{j}(t)
$$

Now $\lambda_{j}\left(t_{0}\right)=0$, so $v_{t}$ could potentially blow up as $t \rightarrow t_{0}$. However, this is prevented by the fact that $g \in D^{\perp}$, which ensures that $v_{t}$ may be written as

$$
v_{t}=-\sum_{j \in I}\left[\int_{\partial \Omega} g \frac{\varphi_{j}(t)-\varphi_{j}\left(t_{0}\right)}{\lambda_{j}(t)-\lambda_{j}\left(t_{0}\right)} d S\right] \varphi_{j}(t) .
$$


Since $\lambda_{j}$ and $\varphi_{j}$ are real-analytic, one has

$$
\lambda_{j}(t)-\lambda_{j}\left(t_{0}\right)=\mu_{j}(t)\left(t-t_{0}\right), \quad \varphi_{j}(t)-\varphi_{j}\left(t_{0}\right)=\psi_{j}(t)\left(t-t_{0}\right),
$$

where $\mu_{j}$ and $\psi_{j}$ are real-analytic near $t_{0}$ with $\psi_{j}$ taking values in $H^{1}(\Omega)$, and $\mu_{j}\left(t_{0}\right)=$ $\lambda_{j}^{\prime}\left(t_{0}\right)>0$. Thus

$$
v_{t}=-\sum_{j \in I}\left[\int_{\partial \Omega} g \frac{\psi_{j}(t)}{\mu_{j}(t)} d S\right] \varphi_{j}(t)
$$

where $\mu_{j}(t) \geq c>0$ near $t_{0}$. The map $[0,1] \rightarrow L^{2}(\partial \Omega),\left.t \mapsto \psi_{j}(t)\right|_{\partial \Omega}$ is real-analytic since the trace operator is bounded from $H^{1}(\Omega)$ to $L^{2}(\partial \Omega)$. Thus $t \mapsto v_{t}$ is realanalytic near $t_{0}$, and one has $\left\|v_{t}\right\|_{H^{1}(\Omega)} \leq C\|g\|_{L^{2}(\partial \Omega)}$ near $t_{0}$. Combined with part (b), this implies that

$$
\left\|u_{t}\right\|_{H^{1}(\Omega)} \leq C\|g\|_{L^{2}(\partial \Omega)} \text { for } t \text { close to } t_{0} .
$$

Next we define $u_{t_{0}}$ so that the map $s \mapsto u_{s}$ is Lipschitz continuous at $t_{0}$. Recalling the operator $D\left(q\left(t_{0}\right)\right)^{\perp} \rightarrow H^{1}(\Omega), g \mapsto u_{g}$, where $u_{g}=T_{q\left(t_{0}\right)}(0, g)$ from Lemma 2.1, we define

$$
u_{t_{0}}:=u_{g}+\sum_{j \in I}\left(v_{t_{0}}-u_{g}, \varphi_{j}\left(t_{0}\right)\right)_{L^{2}(\Omega)} \varphi_{j}\left(t_{0}\right) .
$$

Then $u_{t_{0}}$ solves $\left(\Delta+k^{2} q\left(t_{0}\right)\right) u_{t_{0}}=0$ in $\Omega$ with $\left.\partial_{\nu} u_{t_{0}}\right|_{\partial \Omega}=g$, and one has $P_{t_{0}} u_{t_{0}}=v_{t_{0}}$.

It remains to prove that $s \mapsto u_{s}$ is Lipschitz continuous at $t_{0}$. Note that

$$
\left(\Delta+k^{2} q\left(t_{0}\right)\right)\left(u_{t}-u_{t_{0}}\right)=-k^{2}\left(q(t)-q\left(t_{0}\right)\right) u_{t}=-k^{2}\left(t-t_{0}\right)\left(q_{2}-q_{1}\right) u_{t}
$$

and $\left.\partial_{\nu}\left(u_{t}-u_{t_{0}}\right)\right|_{\partial \Omega}=0$. It follows from Lemma 2.1 that the function $-k^{2}\left(t-t_{0}\right)\left(q_{2}-\right.$ $\left.q_{1}\right) u_{t}$ is in $N\left(q\left(t_{0}\right)\right)^{\perp}$ and that

$$
u_{t}-u_{t_{0}}=T_{q\left(t_{0}\right)}\left(-k^{2}\left(t-t_{0}\right)\left(q_{2}-q_{1}\right) u_{t}, 0\right)+\varphi
$$

for some $\varphi \in N\left(q\left(t_{0}\right)\right)$. Define the operator

$$
G\left(q\left(t_{0}\right)\right): N\left(q\left(t_{0}\right)\right)^{\perp} \rightarrow H^{1}(\Omega), \quad F \mapsto Q_{t_{0}} T_{q\left(t_{0}\right)}(F, 0) .
$$

Then $G\left(q\left(t_{0}\right)\right)$ is bounded (since $Q_{t_{0}}=\mathrm{Id}-P_{t_{0}}$ is bounded on $H^{1}(\Omega)$ ), and

$$
u_{t}-u_{t_{0}}=-k^{2}\left(t-t_{0}\right) G\left(q\left(t_{0}\right)\right)\left(\left(q_{2}-q_{1}\right) u_{t}\right)+P_{t_{0}}\left(u_{t}-u_{t_{0}}\right) .
$$

Using the uniform bound (2.9), we get that

$$
\left\|u_{t}-u_{t_{0}}\right\|_{H^{1}(\Omega)} \leq C\|g\|_{L^{2}(\partial \Omega)}\left|t-t_{0}\right|+\left\|P_{t_{0}}\left(u_{t}-u_{t_{0}}\right)\right\|_{H^{1}(\Omega)} .
$$

To analyze the last term, we note that by the assumption that $g \in D^{\perp}$ and by $(2.7)$ and (2.8)

$$
\begin{aligned}
& P_{t_{0}}\left(u_{t}-u_{t_{0}}\right)=\sum_{j \in I}\left[\int_{\Omega}\left(u_{t}-u_{t_{0}}\right) \varphi_{j}\left(t_{0}\right) d x\right] \varphi_{j}\left(t_{0}\right) \\
& =\sum_{j \in I}\left[\int_{\Omega}\left(u_{t} \varphi_{j}(t)-u_{t_{0}} \varphi_{j}\left(t_{0}\right)\right) d x\right] \varphi_{j}\left(t_{0}\right)-\sum_{j \in I}\left[\int_{\Omega} u_{t}\left(\varphi_{j}(t)-\varphi_{j}\left(t_{0}\right)\right) d x\right] \varphi_{j}\left(t_{0}\right) \\
& =-\sum_{j \in I}\left[\int_{\partial \Omega} g\left[\frac{\psi_{j}(t)}{\mu_{j}(t)}-\frac{\psi_{j}\left(t_{0}\right)}{\mu_{j}\left(t_{0}\right)}\right] d S\right] \varphi_{j}\left(t_{0}\right)-\left(t-t_{0}\right) \sum_{j \in I}\left[\int_{\Omega} u_{t} \psi_{j}(t) d x\right] \varphi_{j}\left(t_{0}\right),
\end{aligned}
$$


where $\mu_{j}$ and $\psi_{j}$ are real-analytic near $t_{0}$ with $\psi_{j}$ taking values in $H^{1}(\Omega)$. Thus in particular

$$
\left\|P_{t_{0}}\left(u_{t}-u_{t_{0}}\right)\right\|_{H^{1}(\Omega)} \leq C\left(\|g\|_{L^{2}(\partial \Omega)}+\left\|u_{t}\right\|_{L^{2}(\Omega)}\right)\left|t-t_{0}\right|
$$

Using (2.9) again, this concludes the proof that $s \mapsto u_{s}$ is Lipschitz continuous near $t_{0}$. Since this is true near $t_{1}, \ldots, t_{K}$, and since $s \mapsto u_{s}$ is real-analytic away from $\left\{t_{1}, \ldots, t_{K}\right\}$, we have proved $(\mathrm{d})$.

We are now ready to prove Theorem 1.1 .

Proof of Theorem 1.1. We will do the proof in three steps.

Step 1: Definition of a finite-dimensional space $D$. We can assume that $q_{2} \geq q_{1}$ and $q_{2} \not \equiv q_{1}$, since the case $q_{2} \equiv q_{1}$ is immediate. Write $d_{1}=d\left(q_{1}\right), d_{2}=d\left(q_{2}\right)$ and $N=d_{2}-d_{1}$, and let $q(t)$ and $\lambda_{j}(t)$ be as in Lemma 2.2. Now the positive Neumann eigenvalues of $q(0)$ are $\lambda_{1}(0), \ldots, \lambda_{d_{1}}(0)$. Since the functions $\lambda_{j}(t)$ are strictly increasing, the positive Neumann eigenvalues related to $q(1)$ are $\lambda_{1}(1), \ldots, \lambda_{d_{1}}(1), \lambda_{j_{1}}(1), \ldots$, $\lambda_{j_{N}}$ (1) for some indices $j_{1}, \ldots, j_{N}$. (Here it is possible that $N=0$.) We reorder the indices for $j \geq d_{1}+1$ so that the positive Neumann eigenvalues related to $q(1)$ are in descending order $\lambda_{1}(1), \ldots, \lambda_{d_{1}}(1), \lambda_{d_{1}+1}(1), \ldots, \lambda_{d_{2}}(1)$. It follows that $\lambda_{j}(t)$ for $j \leq d_{1}$ are positive on $[0,1], \lambda_{j}(t)$ for $d_{1}+1 \leq j \leq d_{2}$ have a unique zero and cross from negative to positive on $[0,1]$, and $\lambda_{j}(t)$ for $j \geq d_{2}+1$ are always negative on $[0,1]$.

Let $0<t_{1}<\cdots<t_{K}<1$ be the times when 0 is a Neumann eigenvalue of $H(t)$, and let

$$
D=D\left(q\left(t_{1}\right)\right) \oplus \cdots \oplus D\left(q\left(t_{K}\right)\right)
$$

as in Lemma 2.3. By Lemmas 2.1 and 2.2, $\operatorname{dim}\left(D\left(q\left(t_{l}\right)\right)\right)$ is the multiplicity of 0 as a Neumann eigenvalue of $H\left(t_{l}\right)$, which is precisely the number of functions $\lambda_{j}(t)$ that vanish at $t_{l}$. Since exactly $N=d_{2}-d_{1}$ functions $\lambda_{j}$ have a zero in [0,1], it follows that $\operatorname{dim}(D) \leq N$. (The dimension of $D$ would be equal to $N$ if all the spaces $D\left(q\left(t_{1}\right)\right), \ldots, D\left(q\left(t_{K}\right)\right)$ would be linearly independent, but this may not be true in general.)

Step 2: We will next show that

$$
\left(\left(\Lambda\left(q_{2}\right)-\Lambda\left(q_{1}\right)\right) g, g\right) \geq 0 \quad \text { for all } g \in D^{\perp} .
$$

Fix $g \in D^{\perp}$, and let $[0,1] \rightarrow H^{1}(\Omega), t \mapsto u_{t}$ be the map in Lemma 2.3. Since $q(0)=q_{1}$ and $q(1)=q_{2}$, it follows that

$$
\left(\left(\Lambda\left(q_{2}\right)-\Lambda\left(q_{1}\right)\right) g, g\right)=\int_{\partial \Omega} u_{1} g d S-\int_{\partial \Omega} u_{0} g d S .
$$

We write, for $t \in[0,1]$,

$$
F(t):=\int_{\partial \Omega} u_{t} g d S=\int_{\Omega}\left(\left|\nabla u_{t}\right|^{2}-k^{2} q(t) u_{t}^{2}\right) d x
$$

Then $F$ is Lipschitz continuous in $[0,1]$ since $t \mapsto u_{t}$ is Lipschitz:

$$
\left|F(t)-F\left(t_{0}\right)\right| \leq\left\|u_{t}-u_{t_{0}}\right\|_{L^{2}(\partial \Omega)}\|g\|_{L^{2}(\partial \Omega)} \leq C_{g}\left\|u_{t}-u_{t_{0}}\right\|_{H^{1}(\Omega)} \leq C_{g}\left|t-t_{0}\right| .
$$

We compute the derivative of $F$ using the fact from Lemma 2.3 that $u_{t}$ is realanalytic in $[0,1] \backslash\left\{t_{1}, \ldots, t_{K}\right\}$, and $\partial_{t} u_{t}$ is the unique solution of

$$
\left(\Delta+k^{2} q(t)\right) \partial_{t} u_{t}=-k^{2} q^{\prime}(t) u_{t} \text { in } \Omega,\left.\quad \partial_{\nu} \partial_{t} u_{t}\right|_{\partial \Omega}=0
$$


Thus

$$
\begin{aligned}
F^{\prime}(t) & =2 \int_{\Omega}\left(\nabla u_{t} \cdot \nabla \partial_{t} u_{t}-k^{2} q(t) u_{t} \partial_{t} u_{t}\right) d x-k^{2} \int_{\Omega} q^{\prime}(t) u_{t}^{2} d x \\
& =2 k^{2} \int_{\Omega} q^{\prime}(t) u_{t}^{2} d x-k^{2} \int_{\Omega} q^{\prime}(t) u_{t}^{2} d x \\
& =k^{2} \int_{\Omega} q^{\prime}(t) u_{t}^{2} d x
\end{aligned}
$$

Since $F(t)$ is Lipschitz continuous and hence absolutely continuous, we may use the fundamental theorem of calculus to compute

$$
\begin{aligned}
\left(\left(\Lambda\left(q_{2}\right)-\Lambda\left(q_{1}\right)\right) g, g\right) & =F(1)-F(0)=\int_{0}^{1} F^{\prime}(t) d t \\
& =k^{2} \int_{0}^{1} \int_{\Omega}\left(q_{2}-q_{1}\right) u_{t}^{2} d x d t .
\end{aligned}
$$

Since $q_{2}-q_{1} \geq 0$ a.e., we get that $\left(\left(\Lambda\left(q_{2}\right)-\Lambda\left(q_{1}\right)\right) g, g\right) \geq 0$ for $g \in D^{\perp}$ as required.

Step 3: One has $d\left(q_{1}, q_{2}\right) \leq d\left(q_{2}\right)-d\left(q_{1}\right)$.

By the previous step one has $\left(\left(\Lambda\left(q_{2}\right)-\Lambda\left(q_{1}\right)\right) g, g\right) \geq 0$ for all $g \in D^{\perp}$. By [24, Corollary 3.3] this implies that $\Lambda\left(q_{1}\right) \leq_{\operatorname{dim}(D)} \Lambda\left(q_{2}\right)$, i.e., that $\Lambda\left(q_{2}\right)-\Lambda\left(q_{1}\right)$ has $\leq \operatorname{dim}(D) \leq d\left(q_{2}\right)-d\left(q_{1}\right)$ negative eigenvalues.

3. Lower bounds for the number of negative eigenvalues. In this section we will prove Theorems 1.3 and 1.4. We will work under the assumption that $q_{2}-q_{1}$ is a positive constant, which ensures that the Neumann eigenvalues and eigenfunctions of $\Delta+k^{2} q(t)$ behave in a very simple way as $t$ varies. (In particular, analytic perturbation theory is not required.)

Proof of Theorem 1.3. The proof proceeds in several steps.

Step 1: Notation for eigenvalues and eigenfunctions. Let $d_{1}=d\left(q_{1}\right)$, and let

$$
\lambda_{1} \geq \lambda_{2} \geq \cdots \geq \lambda_{d_{1}}>0>\lambda_{d_{1}+1} \geq \cdots \rightarrow-\infty
$$

be the Neumann eigenvalues of $\Delta+k^{2} q_{1}$ in $\Omega$. (Here it is possible that $d_{1}=0$, and all eigenvalues are negative.) Let $\left(\varphi_{j}\right)_{j=1}^{\infty}$ be a corresponding orthonormal basis of $L^{2}(\Omega)$ consisting of Neumann eigenfunctions, i.e.,

$$
\left(\Delta+k^{2} q_{1}\right) \varphi_{j}=\lambda_{j} \varphi_{j} \text { in } \Omega,\left.\quad \partial_{\nu} \varphi_{j}\right|_{\partial \Omega}=0 .
$$

Define the potentials $q(t)=(1-t) q_{1}+t q_{2}$. Since by assumption $c=q_{2}-q_{1}$ is a positive constant, we have

$$
q(t)=q_{1}+t c .
$$

Now, one has

$$
\left(\Delta+k^{2} q_{1}\right) \varphi=\lambda \varphi \quad \Longleftrightarrow \quad\left(\Delta+k^{2} q(t)\right) \varphi=\left(\lambda+k^{2} c t\right) \varphi .
$$

Thus the Neumann eigenvalues of $\Delta+k^{2} q(t)$ are given by

$$
\lambda_{j}(t)=\lambda_{j}+k^{2} c t,
$$


and the corresponding $L^{2}$-orthonormal Neumann eigenfunctions $\varphi_{j}(t)=\varphi_{j}$ are independent of $t$. We note that the functions $\lambda_{j}(t), t \in[0,1]$, are strictly increasing. They are positive if $j \leq d_{1}$, cross from negative to positive and satisfy $\lambda_{j}\left(t_{j}\right)=0$ at times

$$
0<t_{d_{1}+1} \leq \cdots \leq t_{d_{2}}<1
$$

if $d_{1}+1 \leq j \leq d_{2}$, and stay negative if $j \geq d_{2}+1$. Here $d_{2}=d\left(q_{2}\right)$.

Step 2: Formula for $\left(\left(\Lambda\left(q_{2}\right)-\Lambda\left(q_{1}\right)\right) g, g\right)$. Fix $g \in L^{2}(\partial \Omega)$, and let $t \in\left[0, t_{d_{1}+1}\right) \cup$ $\left(t_{d_{2}}, 1\right]$. Let $u_{t}$ be the solution of

$$
\left(\Delta+k^{2} q(t)\right) u_{t}=0 \text { in } \Omega,\left.\quad \partial_{\nu} u_{t}\right|_{\partial \Omega}=g .
$$

Note that the Neumann problem is well-posed for $t$ in this range, and as in Lemma 2.1 one has the $L^{2}(\Omega)$-convergent representation

$$
u_{t}=\sum_{j=1}^{\infty} c_{j}(t) \varphi_{j}
$$

with

$$
c_{j}(t)=\int_{\Omega} u_{t} \varphi_{j} d x=-\frac{1}{\lambda_{j}(t)} \int_{\partial \Omega} g \varphi_{j} d S .
$$

As in Lemma 2.3 (but with slightly different notation), we write $u_{t}=v_{t}+w_{t}$, where

$$
v_{t}=\sum_{j=d_{1}+1}^{d_{2}} c_{j}(t) \varphi_{j}, \quad w_{t}=\sum_{j \notin\left[d_{1}+1, d_{2}\right]} c_{j}(t) \varphi_{j} .
$$

Thus we have

$$
\begin{aligned}
& \left(\left(\Lambda\left(q_{2}\right)-\Lambda\left(q_{1}\right)\right) g, g\right)=\int_{\partial \Omega}\left(u_{1}-u_{0}\right) g d S \\
& =\sum_{j=d_{1}+1}^{d_{2}}\left(c_{j}(1)-c_{j}(0)\right) \int_{\partial \Omega} g \varphi_{j} d S+\int_{\partial \Omega}\left(w_{1}-w_{0}\right) g d S \\
& =\sum_{j=d_{1}+1}^{d_{2}} \frac{k^{2} c}{\lambda_{j}(1) \lambda_{j}(0)}\left(\int_{\partial \Omega} g \varphi_{j} d S\right)^{2}+\int_{\partial \Omega}\left(w_{1}-w_{0}\right) g d S .
\end{aligned}
$$

Note that the coefficient $\frac{k^{2} c}{\lambda_{j}(1) \lambda_{j}(0)}$ is negative exactly when $d_{1}+1 \leq j \leq d_{2}$, so that the sum in (3.4) is $\leq 0$ while the last integral may be positive.

Step 3: Formula for $\left(\left(\Lambda\left(q_{2}-b\right)-\Lambda\left(q_{1}+a\right)\right) g, g\right)$. We will now replace $q_{1}$ by $q_{1}+a$ and $q_{2}$ by $q_{2}-b$ and show that for suitable choices of $a$ and $b$, the negative contributions in (3.4) dominate the positive ones. This will imply that the corresponding quadratic form is negative on some finite-dimensional space, yielding a lower bound for the number of negative eigenvalues. We do the rescalings

$$
a=c \alpha, \quad b=c(1-\beta),
$$

where $\alpha, \beta \in[0,1]$ and

$$
q(\alpha)=q_{1}+a, \quad q(\beta)=q_{2}-b .
$$


Equation (3.4) now becomes

$$
\begin{aligned}
\left(\left(\Lambda\left(q_{2}-b\right)-\Lambda\left(q_{1}+a\right)\right) g, g\right)=\sum_{j=d_{1}+1}^{d_{2}} \frac{k^{2} c(\beta-\alpha)}{\lambda_{j}(\beta) \lambda_{j}(\alpha)}\left(\int_{\partial \Omega} g \varphi_{j} d S\right)^{2} & \\
& +\int_{\partial \Omega}\left(w_{\beta}-w_{\alpha}\right) g d S .
\end{aligned}
$$

In the notation of Theorem 1.3, one has $\mu_{1}=\lambda_{d_{1}+1}$ and $\mu_{2}=\lambda_{d_{2}}(1)=\lambda_{d_{2}}+k^{2} c$. Then $t_{d_{1}+1}=c^{-1} k^{-2}\left|\mu_{1}\right|$ (since $\lambda_{d_{1}+1}\left(t_{d_{1}+1}\right)=0$ ) and $t_{d_{2}}=1-c^{-1} k^{-2} \mu_{2}$ (since $\left.\lambda_{d_{2}}\left(t_{d_{2}}\right)=0\right)$. It follows that

$$
\left\{\begin{array}{c}
a \in\left[0, k^{-2}\left|\mu_{1}\right|\right) \text { if and only if } \alpha \in\left[0, t_{d_{1}+1}\right), \\
b \in\left[0, k^{-2} \mu_{2}\right) \text { if and only if } \beta \in\left(t_{d_{2}}, 1\right] .
\end{array}\right.
$$

The next step is to show that the last integral in (3.5) is uniformly bounded over $\alpha \in\left[0, t_{d_{1}+1}\right)$ and $\beta \in\left(t_{d_{2}}, 1\right]$. This will follow since $w_{t}$ is related only to those eigenfrequencies that are uniformly bounded away from zero.

Step 4: $\left\|w_{t}\right\|_{H^{1}(\Omega)} \leq C\|g\|_{L^{2}(\partial \Omega)}$ uniformly over $t \in\left[0, t_{d_{1}+1}\right) \cup\left(t_{d_{2}}, 1\right]$. This follows directly from Lemma 2.3(c).

Step 5: Proof of part (a). We will show that there is a subspace $V$ of $L^{2}(\partial \Omega)$ with $\operatorname{dim}(V)=N_{1}$ such that (3.5) is negative when $g \in V \backslash\{0\}, \alpha<t_{d_{1}+1}$ is close to $t_{d_{1}+1}$, and $\beta \in\left(t_{d_{2}}, 1\right]$. Combined with (3.6) and [24, Lemma 3.2(b)] applied to $A=-\left(\Lambda\left(q_{2}-b\right)-\Lambda\left(q_{1}+a\right)\right)$ with $r=0$, this will prove part (a).

By the trace theorem and Step 4, we have

$$
\left|\int_{\partial \Omega}\left(w_{\beta}-w_{\alpha}\right) g d S\right| \leq C\left\|w_{\beta}-w_{\alpha}\right\|_{H^{1}(\Omega)}\|g\|_{L^{2}(\partial \Omega)} \leq C\|g\|_{L^{2}(\partial \Omega)}^{2}
$$

uniformly over $\alpha \in\left[0, t_{d_{1}+1}\right)$ and $\beta \in\left(t_{d_{2}}, 1\right]$. Thus

$$
\left(\left(\Lambda\left(q_{2}-b\right)-\Lambda\left(q_{1}+a\right)\right) g, g\right) \leq \sum_{j=d_{1}+1}^{d_{2}} \frac{k^{2} c(\beta-\alpha)}{\lambda_{j}(\beta) \lambda_{j}(\alpha)}\left(\int_{\partial \Omega} g \varphi_{j} d S\right)^{2}+C\|g\|_{L^{2}(\partial \Omega)}^{2} .
$$

If $j \in\left[d_{1}+1, d_{2}\right]$, then $\alpha<t_{j}$ and $\beta>t_{j}$, and $\lambda_{j}(\beta)=\lambda_{j}\left(t_{j}\right)+k^{2} c\left(\beta-t_{j}\right)=$ $k^{2} c\left(\beta-t_{j}\right)>0$. Thus one has

$$
\frac{k^{2} c(\beta-\alpha)}{\lambda_{j}(\beta)} \geq \frac{k^{2} c\left(\beta-t_{j}\right)}{k^{2} c\left(\beta-t_{j}\right)}=1 .
$$

Since $\lambda_{j}(\alpha)=\lambda_{j}\left(t_{j}\right)-k^{2} c\left(t_{j}-\alpha\right)=-k^{2} c\left(t_{j}-\alpha\right)<0$, we obtain that

$$
\begin{aligned}
&\left(\left(\Lambda\left(q_{2}-b\right)-\Lambda\left(q_{1}+a\right)\right) g, g\right) \leq-\sum_{j=d_{1}+1}^{d_{2}} \frac{1}{k^{2} c\left(t_{j}-\alpha\right)}\left(\int_{\partial \Omega} g \varphi_{j} d S\right)^{2} \\
&+C\|g\|_{L^{2}(\partial \Omega)}^{2}
\end{aligned}
$$

uniformly over $\alpha \in\left[0, t_{d_{1}+1}\right)$ and $\beta \in\left(t_{d_{2}}, 1\right]$.

Recall now the assumption that $\lambda_{d_{1}+1}$ has multiplicity $N_{1}$, and define

$$
V=\operatorname{span}\left\{\left.\varphi_{d_{1}+1}\right|_{\partial \Omega}, \ldots,\left.\varphi_{d_{1}+N_{1}}\right|_{\partial \Omega}\right\} .
$$


Here $\varphi_{d_{1}+1}, \ldots, \varphi_{d_{1}+N_{1}}$ are Neumann eigenfunctions corresponding to $\lambda_{d_{1}+1}$. We claim that $\operatorname{dim}(V)=N_{1}$. For if $\left.a_{1} \varphi_{d_{1}+1}\right|_{\partial \Omega}+\cdots+\left.a_{N_{1}} \varphi_{d_{1}+N_{1}}\right|_{\partial \Omega}=0$, then the function $\varphi=a_{1} \varphi_{d_{1}+1}+\cdots+a_{N_{1}} \varphi_{d_{1}+N_{1}}$ satisfies

$$
\left(\Delta+k^{2} q_{1}\right) \varphi=\lambda_{d_{1}+1} \varphi \text { in } \Omega,\left.\quad \varphi\right|_{\partial \Omega}=\left.\partial_{\nu} \varphi\right|_{\partial \Omega}=0 .
$$

By the unique continuation principle this implies that $\varphi \equiv 0$, and since $\left\{\varphi_{j}\right\}$ are orthonormal in $L^{2}(\Omega)$ we obtain $a_{1}=\cdots=a_{N_{1}}=0$. This proves that $\operatorname{dim}(V)=N_{1}$.

Let now $g \in V \backslash\{0\}$. Since $\lambda_{d_{1}+1}$ has multiplicity $N_{1}$ and since $t_{j}$ is the unique zero of $t \mapsto \lambda_{j}(t)$, by (3.1) one has $t_{d_{1}+1}=\cdots=t_{d_{1}+N_{1}}$, and thus

$$
\begin{aligned}
\left(\left(\Lambda\left(q_{2}-b\right)-\Lambda\left(q_{1}+a\right)\right) g, g\right) & \leq-\frac{1}{k^{2} c\left(t_{d_{1}+1}-\alpha\right)} \sum_{j=d_{1}+1}^{d_{1}+N_{1}}\left(\int_{\partial \Omega} g \varphi_{j} d S\right)^{2} \\
& -\sum_{j=d_{1}+N_{1}+1}^{d_{2}} \frac{1}{k^{2} c\left(t_{j}-\alpha\right)}\left(\int_{\partial \Omega} g \varphi_{j} d S\right)^{2}+C\|g\|_{L^{2}(\partial \Omega)}^{2}
\end{aligned}
$$

uniformly over $\alpha \in\left[0, t_{d_{1}+1}\right)$ and $\beta \in\left(t_{d_{2}}, 1\right]$. The middle term on the right is $\leq 0$, and writing

$$
\delta=\inf _{g \in V,\|g\|_{L^{2}(\partial \Omega)}} \sum_{j=d_{1}+1}^{d_{1}+N_{1}}\left(\int_{\partial \Omega} g \varphi_{j} d S\right)^{2},
$$

where $\delta>0$ (the infimum is over the unit sphere in a finite-dimensional normed space and the quantity inside the infimum is positive for $g \in V \backslash\{0\}$ ), we obtain that

$$
\left(\left(\Lambda\left(q_{2}-b\right)-\Lambda\left(q_{1}+a\right)\right) g, g\right) \leq\left(-\frac{\delta}{k^{2} c\left(t_{d_{1}+1}-\alpha\right)}+C\right)\|g\|_{L^{2}(\partial \Omega)}^{2},
$$

where $C$ is uniform over $\alpha \in\left[0, t_{d_{1}+1}\right)$ and $\beta \in\left(t_{d_{2}}, 1\right]$. Thus choosing $\alpha<t_{d_{1}+1}$ sufficiently close to $t_{d_{1}+1}$, one has $\left(\left(\Lambda\left(q_{2}-b\right)-\Lambda\left(q_{1}+a\right)\right) g, g\right)<0$ for $g \in V \backslash\{0\}$. This concludes the proof of part (a).

Step 6: Proof of part (b). This is completely analogous to Step 5: one defines the subspace

$$
V=\operatorname{span}\left\{\left.\varphi_{d_{2}-N_{2}+1}\right|_{\partial \Omega}, \ldots,\left.\varphi_{d_{2}}\right|_{\partial \Omega}\right\}
$$

and shows that $\left(\left(\Lambda\left(q_{2}-b\right)-\Lambda\left(q_{1}+a\right)\right) g, g\right)<0$ for $g \in V \backslash\{0\}$ when $\beta \in\left(t_{d_{2}}, 1\right]$ is sufficiently close to $t_{d_{2}}$.

Proof of Theorem 1.4. (a) If $\lambda_{j}$ are the Neumann eigenvalues of $\Delta+k^{2} q$ in $\Omega$, then $\lambda_{j} \pm k^{2} \varepsilon$ are the eigenvalues of $\Delta+k^{2}(q \pm \varepsilon)$ in $\Omega$. Thus if $\varepsilon_{0}>0$ is small enough and $\varepsilon \leq \varepsilon_{0}$, one has $d(q+\varepsilon)-d(q-\varepsilon)=N$, and $\Lambda(q+\varepsilon)-\Lambda(q-\varepsilon)$ has at most $N$ negative eigenvalues by Theorem 1.1. Moreover, by Theorem 1.3 with $q_{1}=q-\varepsilon_{0}$, $q_{2}=q+\varepsilon_{0}, \mu_{1}=-k^{2} \varepsilon_{0}$, and $\mu_{2}=k^{2} \varepsilon_{0}$, we obtain that $\Lambda(q+\varepsilon)-\Lambda(q-\varepsilon)$ has at least $N$ negative eigenvalues for $\varepsilon$ small.

(b) Recall that we now assume that $\Omega:=(0,1)^{2}$. It is enough to show that for any even $N \geq 2$, there is an eigenvalue $\mu$ of $\Delta$ in $\Omega$ with multiplicity $N$. If this holds, then choosing $c=-k^{-2} \mu$ gives that 0 is an eigenvalue of $\Delta+k^{2} c$ of multiplicity $N$, and the result follows from part (a).

An orthonormal basis of $L^{2}(\Omega)$ consisting of Neumann eigenfunctions of $\Delta$ in $\Omega$ is given by $\left(\varphi_{l_{1}, l_{2}}\right)_{l_{1}, l_{2}=0}^{\infty}$, where

$$
\varphi_{l_{1}, l_{2}}(x)=c_{l_{1}, l_{2}} \cos \left(l_{1} x_{1}\right) \cos \left(l_{2} x_{2}\right)
$$


for some normalizing constants $c_{l_{1}, l_{2}}$. The eigenvalue corresponding to $\varphi_{l_{1}, l_{2}}$ is $-\left(l_{1}^{2}+\right.$ $\left.l_{2}^{2}\right)$. See, e.g., [11].

We set $\lambda=5^{r}$, where $r \geq 1$ is an odd integer, and write $N=r+1$. Since 5 is a prime of the form $4 m+1$, there are $4 N$ pairs $\left(s_{1}, s_{2}\right) \in \mathbb{Z}^{2}$ such that $\lambda=s_{1}^{2}+s_{2}^{2}[13$, Theorem 278]. Now $r$ is odd, so $\lambda$ is not a square and both $s_{1}$ and $s_{2}$ must be nonzero, and thus there are exactly $N$ pairs $\left(l_{1}, l_{2}\right) \in(\mathbb{N} \cup\{0\})^{2}$ so that $\lambda=l_{1}^{2}+l_{2}^{2}$. This shows that the multiplicity of $-\lambda$ as a Neumann eigenvalue of $\Delta$ is exactly $N$.

4. The Helmholtz equation with constant parameter. In this section, we will treat the Neumann problem for the Helmholtz equation

$$
\left\{\begin{aligned}
\left(\Delta+k^{2} q\right) u & =0 \text { in } \Omega, \\
\partial_{\nu} u & =g \text { on } \partial \Omega
\end{aligned}\right.
$$

with a constant coefficient $q(x)=$ const. In this case, the Helmholtz solution operator can be expressed using the Neumann eigenfunctions of the Laplace equation, which allows us to give a simple independent proof of Theorem 1.1 and show that the dimension bound in Theorem 1.1 is sharp for the Helmholtz solution operators.

For the special case of a constant coefficient in a two-dimensional unit square we also derive an infinite matrix representation of the ND operator and study numerically the question whether the bound in Theorem 1.1 is sharp for the ND operators.

\subsection{The dimension bound for the constant parameter case.}

Definition 4.1. For $0 \not \equiv q \in L^{\infty}(\Omega)$ and a nonresonant wavenumber $k>0$ we define the Helmholtz solution operator

$$
S(q): H^{1}(\Omega) \rightarrow H^{1}(\Omega), F \mapsto v,
$$

where $v \in H^{1}(\Omega)$ solves

$$
\int_{\Omega}\left(\nabla v \cdot \nabla w-k^{2} q v w\right) d x=(F, w)_{H^{1}(\Omega)} \quad \text { for all } w \in H^{1}(\Omega) .
$$

Note that the ND operator

$$
\Lambda(q): L^{2}(\partial \Omega) \rightarrow L^{2}(\partial \Omega),\left.\quad g \mapsto u\right|_{\partial \Omega},
$$

where $u \in H^{1}(\Omega)$ solves (4.1), obviously fulfills

$$
\Lambda(q)=\gamma S(q) \gamma^{*}
$$

where $\gamma$ denotes the compact trace operator

$$
\gamma: H^{1}(\Omega) \rightarrow L^{2}(\partial \Omega),\left.\quad v \mapsto v\right|_{\partial \Omega} .
$$

TheOREM 4.2. Let $\Omega \subset \mathbb{R}^{n}$ be a bounded Lipschitz domain. Let $a, b \in \mathbb{R}$ with $a<b$, and let $k>0$ be nonresonant for $q(x)=a$ and $q(x)=b$. Then

(a) $S(b)-S(a)$ has exactly $d(b)-d(a)$ negative eigenvalues,

(b) $\Lambda(b)-\Lambda(a)$ has at most $d(b)-d(a)$ negative eigenvalues.

Note that (b) follows from Theorem 1.1, but our proof of Theorem 4.2 is independent of this result and rather elementary, so we believe that this is of independent interest. 
As in the proof of Lemma 2.1 (see also [24, section 2.1]), we let Id : $H^{1}(\Omega) \rightarrow$ $H^{1}(\Omega)$ denote the identity operator, $\iota: H^{1}(\Omega) \rightarrow L^{2}(\Omega)$ denote the compact embedding, and $M_{q}: L^{2}(\Omega) \rightarrow L^{2}(\Omega)$ denote the multiplication operator by $q$. Then $K:=\iota^{*} \iota$ and $K_{q}:=\iota^{*} M_{q} \iota$ are compact self-adjoint linear operators from $H^{1}(\Omega)$ to $H^{1}(\Omega)$, and

$$
S(q):=\left(\mathrm{Id}-K-k^{2} K_{q}\right)^{-1},
$$

where the inverse exists if and only if $k>0$ is nonresonant for the potential $q$ (cf., e.g., [24, Lemma 2.2]).

For constant coefficients $q(x)=a \in \mathbb{R}$ this simplifies to

$$
K_{a}=a K \quad \text { and } \quad S(a)=\left(\operatorname{Id}-\left(1+a k^{2}\right) K\right)^{-1} .
$$

Since $K: H^{1}(\Omega) \rightarrow H^{1}(\Omega)$ is a compact self-adjoint, positive definite operator, there exists an orthonormal basis $\left(v_{l}\right)_{l \in \mathbb{N}}$ of $H^{1}(\Omega)$ of eigenfunctions corresponding to eigenvalues $\lambda_{l}>0$,

$$
K v_{l}=\lambda_{l} v_{l} \quad \text { for all } l \in \mathbb{N} .
$$

Note that in this section, $\lambda_{l}$ are the eigenvalues of the compact operator $K$ which converge to zero (unlike in the earlier sections, where $\lambda_{j}$ were Neumann eigenvalues converging to $-\infty)$.

LEMMA 4.3.

(a) A function $v \in H^{1}(\Omega)$ is an eigenfunction of $K$ with eigenvalue $\lambda$ if and only if $v$ is a Neumann eigenfunction of the Laplace equation with Neumann eigenvalue $\frac{1}{\lambda}-1$, i.e.,

$$
-\Delta v=\left(\frac{1}{\lambda}-1\right) v,\left.\quad \partial_{\nu} v\right|_{\partial \Omega}=0
$$

(b) $k>0$ is nonresonant for the potential $q(x)=a \in \mathbb{R}$ if and only if

$$
\frac{1}{1+a k^{2}} \notin\left\{\lambda_{1}, \lambda_{2}, \ldots\right\},
$$

i.e., if and only if $a k^{2}$ is not a Neumann eigenvalue. Moreover,

$$
d(a)=\#\left\{\lambda_{l}: \lambda_{l}>\frac{1}{1+a k^{2}}\right\} .
$$

(c) If $k>0$ is nonresonant for the potential $q(x)=a \in \mathbb{R}$, then

$$
S(a) v_{l}=\left(\operatorname{Id}-\left(1+a k^{2}\right) K\right)^{-1} v_{l}=\frac{1}{1-\left(1+a k^{2}\right) \lambda_{l}} v_{l} .
$$

(d) Let $a, b \in \mathbb{R}$ with $a<b$, and $k>0$ be nonresonant for $q(x)=a$ and $q(x)=b$. Then

$$
(F,(S(b)-S(a)) F)_{H^{1}(\Omega)}=\sum_{l=1}^{\infty} c_{l}\left(F, v_{l}\right)_{H^{1}(\Omega)}^{2},
$$

where $0 \neq c_{l} \in \mathbb{R}$, and the number of negative $c_{l}$ is exactly $d(b)-d(a)$. 
Proof. $K v=\lambda v$ is equivalent to

$$
\begin{aligned}
& \int_{\Omega} \nabla v \cdot \nabla w d x-\left(\frac{1}{\lambda}-1\right) \int_{\Omega} v w d x \\
& =((\operatorname{Id}-K) v, w)_{H^{1}(\Omega)}-\left(\frac{1}{\lambda}-1\right)(K v, w)_{H^{1}(\Omega)} \\
& =\left(\left(\operatorname{Id}-\frac{1}{\lambda} K\right) v, w\right)_{H^{1}(\Omega)}=0 \quad \text { for all } w \in H^{1}(\Omega),
\end{aligned}
$$

which is the variational formulation equivalent to

$$
-\Delta v=\left(\frac{1}{\lambda}-1\right) v,\left.\quad \partial_{\nu} v\right|_{\partial \Omega}=0
$$

This proves (a).

The first parts of (b) and (c) are obvious. The second part of (b) has been proved in [24, Lemma 2.1].

To prove (d) note that for all $F \in H^{1}(\Omega)$

$$
F=\sum_{l=1}^{\infty} v_{l}\left(F, v_{l}\right)_{H^{1}(\Omega)},
$$

where the sum is convergent in $H^{1}(\Omega)$. Hence,

$$
\begin{aligned}
& (F,(S(b)-S(a)) F)_{H^{1}(\Omega)} \\
& =\left(F,(S(b)-S(a)) \sum_{l=1}^{\infty} v_{l}\left(F, v_{l}\right)_{H^{1}(\Omega)}\right)_{H^{1}(\Omega)} \\
& =\sum_{l=1}^{\infty}\left(\frac{1}{1-\left(1+b k^{2}\right) \lambda_{l}}-\frac{1}{1-\left(1+a k^{2}\right) \lambda_{l}}\right)\left(F, v_{l}\right)_{H^{1}(\Omega)}^{2} .
\end{aligned}
$$

For the coefficients

$$
\begin{aligned}
c_{l} & :=\frac{1}{1-\left(1+b k^{2}\right) \lambda_{l}}-\frac{1}{1-\left(1+a k^{2}\right) \lambda_{l}} \\
& =\frac{(b-a) k^{2}}{\left(\frac{1}{\lambda_{l}}-\left(1+b k^{2}\right)\right)\left(\frac{1}{\lambda_{l}}-\left(1+a k^{2}\right)\right)}
\end{aligned}
$$

we obviously have that $c_{l} \neq 0$ and that $c_{l}<0$ if and only if

$$
\frac{1}{1+a k^{2}}>\lambda_{l}>\frac{1}{1+b k^{2}} \text {. }
$$

By the second part of (b), the number of negative $c_{l}$ is exactly $d(b)-d(a)$.

Proof of Theorem 4.2. Using Lemma 4.3 we have that

$$
V:=\operatorname{span}\left\{v_{l}: c_{l}<0\right\} \subset H^{1}(\Omega)
$$


is a subspace of dimension $\operatorname{dim}(V)=d(b)-d(a)$,

$$
\begin{array}{ll}
(F,(S(b)-S(a)) F)_{H^{1}(\Omega)}<0 & \text { for all } F \in V \backslash\{0\}, \text { and } \\
(F,(S(b)-S(a)) F)_{H^{1}(\Omega)} \geq 0 & \text { for all } F \perp_{H^{1}} V .
\end{array}
$$

Using, e.g., [24, Lemma 3.2], this shows that $S(b)-S(a)$ has exactly $d(b)-d(a)$ negative eigenvalues and thus proves Theorem 4.2(a).

Using that $\Lambda(b)-\Lambda(a)=\gamma(S(b)-S(a)) \gamma^{*}$, we also have that

$$
\int_{\partial \Omega} g(\Lambda(b)-\Lambda(a)) g d S \geq 0
$$

for all $g$ with $\gamma^{*} g \perp_{H^{1}} V$, which is equivalent to

$$
(g, \gamma v)_{L^{2}(\partial \Omega)}=\left(\gamma^{*} g, v\right)_{H^{1}(\Omega)}=0 \quad \text { for all } v \in V,
$$

and thus

$$
\int_{\partial \Omega} g(\Lambda(b)-\Lambda(a)) g d S \geq 0 \quad \text { for all } g \perp_{L^{2}} \gamma(V) .
$$

Using $\operatorname{dim}(\gamma(V)) \leq \operatorname{dim}(V)=d(b)-d(a)$ and [24, Corollary 3.3]), this shows that $\Lambda(b)-\Lambda(a)$ has at most $d(b)-d(a)$ negative eigenvalues and thus proves Theorem $4.2(\mathrm{~b})$.

4.2. Helmholtz equation on the two-dimensional unit square. We now consider the special case of the Helmholtz equation with constant parameter $q(x)=$ $a \in \mathbb{R}$ on the two-dimensional unit square

$$
\Omega:=(0,1)^{2}
$$

and derive an infinite matrix representation for the ND operator $\Lambda(a)$.

For the unit square the Neumann eigenfunctions are well known.

Lemma 4.4. For $l, m \in \mathbb{N}_{0}$ we define

$$
v_{l, m}: \Omega \rightarrow \mathbb{R}, \quad v_{l, m}(x, y):=d_{l} d_{m} \cos (\pi l x) \cos (\pi m y),
$$

with $d_{0}:=1$ and $d_{j}=\sqrt{2}$ for $j \in \mathbb{N}$. The functions $v_{l, m}$ are Neumann eigenfunctions of the Laplacian

$$
-\Delta v_{l, m}=\pi^{2}\left(l^{2}+m^{2}\right) v_{l, m},\left.\quad \partial_{\nu} v_{l, m}\right|_{\partial \Omega}=0,
$$

and eigenfunctions of $K: H^{1}(\Omega) \rightarrow H^{1}(\Omega)$

$$
K v_{l, m}=\lambda_{l, m} v_{l, m} \quad \text { with } \quad \lambda_{l, m}:=\frac{1}{1+\pi^{2}\left(l^{2}+m^{2}\right)} .
$$

$\left(v_{l, m}\right)_{l, m \in \mathbb{N}}$ is an orthonormal basis of $L^{2}(\Omega)$, and $\left(\sqrt{\lambda_{l, m}} v_{l, m}\right)_{l, m \in \mathbb{N}}$ is an orthonormal basis of $H^{1}(\Omega)$.

Proof. It is easily checked that the functions $\left(v_{l, m}\right)_{l, m \in \mathbb{N}}$ are Neumann eigenfunctions and that they form an orthonormal basis of $L^{2}(\Omega)$. Lemma 4.3 implies that the $\left(v_{l, m}\right)_{l, m \in \mathbb{N}}$ are also eigenfunctions of $K$, and this yields that

$$
\left(v_{l, m}, v_{l^{\prime}, m^{\prime}}\right)_{H^{1}(\Omega)}=\frac{1}{\lambda_{l, m}} \int_{\Omega} v_{l, m} v_{l^{\prime}, m^{\prime}} d x=\frac{1}{\lambda_{l, m}} \delta_{l, l^{\prime}} \delta_{m, m^{\prime}},
$$

which shows that $\left(\sqrt{\lambda_{l, m}} v_{l, m}\right)_{l, m \in \mathbb{N}}$ is an orthonormal basis of $H^{1}(\Omega)$. 
We can now expand the ND operator $\Lambda(a)$ in an orthonormal basis of cosine functions on the four sides of $\partial \Omega$.

Lemma 4.5. Define $g_{s}: \partial \Omega \rightarrow \mathbb{R}\left(s \in \mathbb{N}_{0}\right)$ by setting for all $j \in \mathbb{N}_{0}$

$$
\begin{aligned}
g_{4 j+0}(x, y) & :=d_{j} \cos (j \pi y) \mathbf{1}_{\Sigma_{\text {right }}(x, y),} & \Sigma_{\text {right }} & :=\{1\} \times(0,1), \\
g_{4 j+1}(x, y) & :=d_{j} \cos (j \pi(1-x)) \mathbf{1}_{\Sigma_{\text {top }}}(x, y), & \Sigma_{\text {top }} & :=(0,1) \times\{1\}, \\
g_{4 j+2}(x, y) & :=d_{j} \cos (j \pi(1-y)) \mathbf{1}_{\Sigma_{\text {left }}}(x, y), & \Sigma_{\text {left }} & :=\{0\} \times(0,1), \\
g_{4 j+3}(x, y) & :=d_{j} \cos (j \pi x) \mathbf{1}_{\Sigma_{\text {bottom }}}(x, y), & \Sigma_{\text {bottom }}: & :=(0,1) \times\{0\} .
\end{aligned}
$$

Then $\left(g_{s}\right)_{s \in \mathbb{N}_{0}} \subseteq L^{2}(\partial \Omega)$ is an orthonormal basis of $L^{2}(\partial \Omega)$.

The infinite matrix representation of $\Lambda(a)$ with respect to this basis is given by $4 \times 4$-blocks of the form

$$
\left(\int_{\partial \Omega} g_{4 i+p} \Lambda(a) g_{4 j+r} d s\right)_{p, r=0, \ldots, 3}=\left(\begin{array}{cccc}
M_{0} & M_{1} & M_{2} & M_{3} \\
M_{3} & M_{0} & M_{1} & M_{2} \\
M_{2} & M_{3} & M_{0} & M_{1} \\
M_{1} & M_{2} & M_{3} & M_{0}
\end{array}\right)
$$

where (for $i, j \in \mathbb{N}_{0}$ )

$$
\begin{array}{ll}
M_{0}= \begin{cases}\delta_{i j} \frac{\operatorname{coth}\left(\sqrt{\pi^{2} i^{2}-a k^{2}}\right)}{\sqrt{\pi^{2} i^{2}-a k^{2}}} & \text { for } i^{2}>\frac{a k^{2}}{\pi^{2}}, \\
-\delta_{i j} \frac{\cot \left(\sqrt{a k^{2}-\pi^{2} i^{2}}\right)}{\sqrt{a k^{2}-\pi^{2} i^{2}}} & \text { for } i^{2}<\frac{a k^{2}}{\pi^{2}},\end{cases} \\
M_{2}= \begin{cases}\delta_{i j}(-1)^{i} \frac{\operatorname{csch}\left(\sqrt{\pi^{2} i^{2}-a k^{2}}\right)}{\sqrt{\pi^{2} i^{2}-a k^{2}}} & \text { for } i^{2}>\frac{a k^{2}}{\pi^{2}}, \\
-\delta_{i j}(-1)^{i} \frac{\csc \left(\sqrt{a k^{2}-\pi^{2} i^{2}}\right)}{\sqrt{a k^{2}-\pi^{2} i^{2}}} & \text { for } i^{2}<\frac{a k^{2}}{\pi^{2}},\end{cases}
\end{array}
$$

Proof. Clearly, $\left(g_{s}\right)_{s \in \mathbb{N}_{0}} \subseteq L^{2}(\partial \Omega)$ is an orthonormal basis of $L^{2}(\partial \Omega)$, and

$$
\begin{aligned}
I_{0}(j, l, m) & :=\left.\int_{\partial \Omega} g_{4 j+0} v_{l, m}\right|_{\partial \Omega} d s=(-1)^{l} d_{l} \delta_{j m}, \\
I_{1}(j, l, m) & :=\left.\int_{\partial \Omega} g_{4 j+1} v_{l, m}\right|_{\partial \Omega} d s=(-1)^{m+j} d_{m} \delta_{j l}, \\
I_{2}(j, l, m) & :=\left.\int_{\partial \Omega} g_{4 j+2} v_{l, m}\right|_{\partial \Omega} d s=(-1)^{j} d_{l} \delta_{j m}, \\
I_{3}(j, l, m) & :=\left.\int_{\partial \Omega} g_{4 j+3} v_{l, m}\right|_{\partial \Omega} d s=d_{m} \delta_{j l} .
\end{aligned}
$$

Using that $\left(\sqrt{\lambda_{l m}} v_{l m}\right)_{l, m \in \mathbb{N}}$ is an orthonormal basis of $H^{1}(\Omega)$ that diagonalizes the solution operator (cf. Lemma 4.3(c)) we have that

$$
\begin{aligned}
\int_{\partial \Omega} g_{4 i+p} \Lambda(a) g_{4 j+r} d s & =\left(\gamma^{*} g_{4 i+p}, S(a) \gamma^{*} g_{4 j+r}\right)_{H^{1}(\Omega)} \\
& =\sum_{l, m=0}^{\infty} \frac{\lambda_{l m}}{1-\left(1+a k^{2}\right) \lambda_{l m}} I_{p}(i, l, m) I_{r}(j, l, m) \\
& =\sum_{l, m=0}^{\infty} \frac{1}{\pi^{2}\left(l^{2}+m^{2}\right)-a k^{2}} I_{p}(i, l, m) I_{r}(j, l, m) .
\end{aligned}
$$


The assertion then follows from a simple calculation using the sum formulas

$$
\begin{gathered}
\sum_{m=0}^{\infty} \frac{d_{m}^{2}}{\pi^{2} m^{2}+c}=\left\{\begin{aligned}
-\frac{\cot (\sqrt{-c})}{\sqrt{-c}} & \text { for } c<0, \sqrt{-c} \notin \pi \mathbb{N}, \\
\frac{\operatorname{coth}(\sqrt{c})}{\sqrt{c}} & \text { for } c>0,
\end{aligned}\right. \\
\sum_{m=0}^{\infty}(-1)^{m} \frac{d_{m}^{2}}{\pi^{2} m^{2}+c}=\left\{\begin{aligned}
-\frac{\csc (\sqrt{-c})}{\sqrt{-c}} & \text { for } c<0, \sqrt{-c} \notin \pi \mathbb{N}, \\
\frac{\operatorname{csch}(\sqrt{c})}{\sqrt{c}} & \text { for } c>0
\end{aligned}\right.
\end{gathered}
$$

(see, e.g., [33, Formula (1), p. 327, and Formula (4), p. 329]).

4.3. Numerical evaluation of the dimension bound. We still consider the special case of the Helmholtz equation on the unit square $\Omega=(0,1)^{2}$ with constant parameter $q(x)=a \in \mathbb{R}$, resp., $q(x)=b \in \mathbb{R}$, and fix $k:=1$ without loss of generality. It follows from Lemmas 4.3 and 4.4 that resonances occur when $a$ or $b$ equals $\pi^{2}\left(l^{2}+m^{2}\right)$ with $l, m \in \mathbb{N}_{0}$ and that

$$
d(b)-d(a)=\#\left\{l, m \in \mathbb{N}_{0}: a<\pi^{2}\left(l^{2}+m^{2}\right)<b\right\} .
$$

We know from Theorem 4.2 (and the more general Theorem 1.1) that $\Lambda(b)-$ $\Lambda(a)$ will have at most $d(b)-d(a)$ negative eigenvalues. Moreover, we know from Theorem 1.4 that this bound is achieved, when $a$ and $b$ are sufficiently close together and only slightly smaller, resp., larger than a Neumann eigenvalue $\pi^{2}\left(l^{2}+m^{2}\right)$, and $d(b)-d(a)$ will then be the multiplicity of this Neumann eigenvalue which can attain any even positive integer.

We will now numerically evaluate how the number of negative eigenvalues of $\Lambda(b)-\Lambda(a)$ behaves. To this end we use the numerical programming language MATLAB to calculate a $1000 \times 1000$ matrix approximating $\Lambda(b)-\Lambda(a)$ using the matrix representation formula in Lemma 4.5 for $i, j=0, \ldots, 249$. We estimated the error in this finite-dimensional approximation to be below $\delta=10^{-5}$ in the spectral norm by comparing $\Lambda(b)-\Lambda(a)$ to its upper left $500 \times 500$ entries (filled up by zeros to a $1000 \times 1000$ matrix). Accordingly, we considered eigenvalues below $-\delta$ to be negative and counted their number (with multiplicity).

Figure 1 shows this numerically computed number of negative eigenvalues of $\Lambda(b)-\Lambda(a)$ and the theoretical bound $d(b)-d(a)$ as a function of $b \in \mathbb{R}$ for $a=-10$ (top left), $a=10$ (top right), $a=100$ (bottom left), and $a=200$ (bottom right). Whenever $b$ crosses an eigenvalue $\pi^{2}\left(l^{2}+m^{2}\right)$ with $l, m \in \mathbb{N}_{0}$ the theoretical bound $d(b)-d(a)$ increases by the multiplicity of this eigenvalue. The plots indicate that the number of negative eigenvalue $\Lambda(b)-\Lambda(a)$ also increases by the multiplicity of this eigenvalue but that there is an additional effect decreasing the number of negative eigenvalues when $b-a$ increases.

To further investigate this additional effect, Figure 2 shows the values of the eigenvalues of $\Lambda(b)-\Lambda(a)$ as a function of $b$ for fixed $a=-10$. More precisely, for each integer $b=-10,-9, \ldots, 200$ (excluding the resonance $b=0$ ), the black dots are plotted at the position $\left(b, \lambda_{j}(a, b)\right)$, where $\lambda_{j}(a, b), j=1, \ldots, 1000$, are the numerically calculated eigenvalues of $\Lambda(b)-\Lambda(a)$. The red dashed lines show the positions of the Neumann eigenvalues. Whenever $b$ crosses an eigenvalue, new negative eigenvalues of $\Lambda(b)-\Lambda(a)$ appear. But at the same time the values of the eigenvalues increase with $b-a$, and it seems that negative eigenvalues can become positive again, which would explain the drops in the number of negative eigenvalues observed in Figure 1.

Let us stress however that this numerical experiment is only an indication of what might happen to stimulate further research. We do not have a rigorous proof that the 

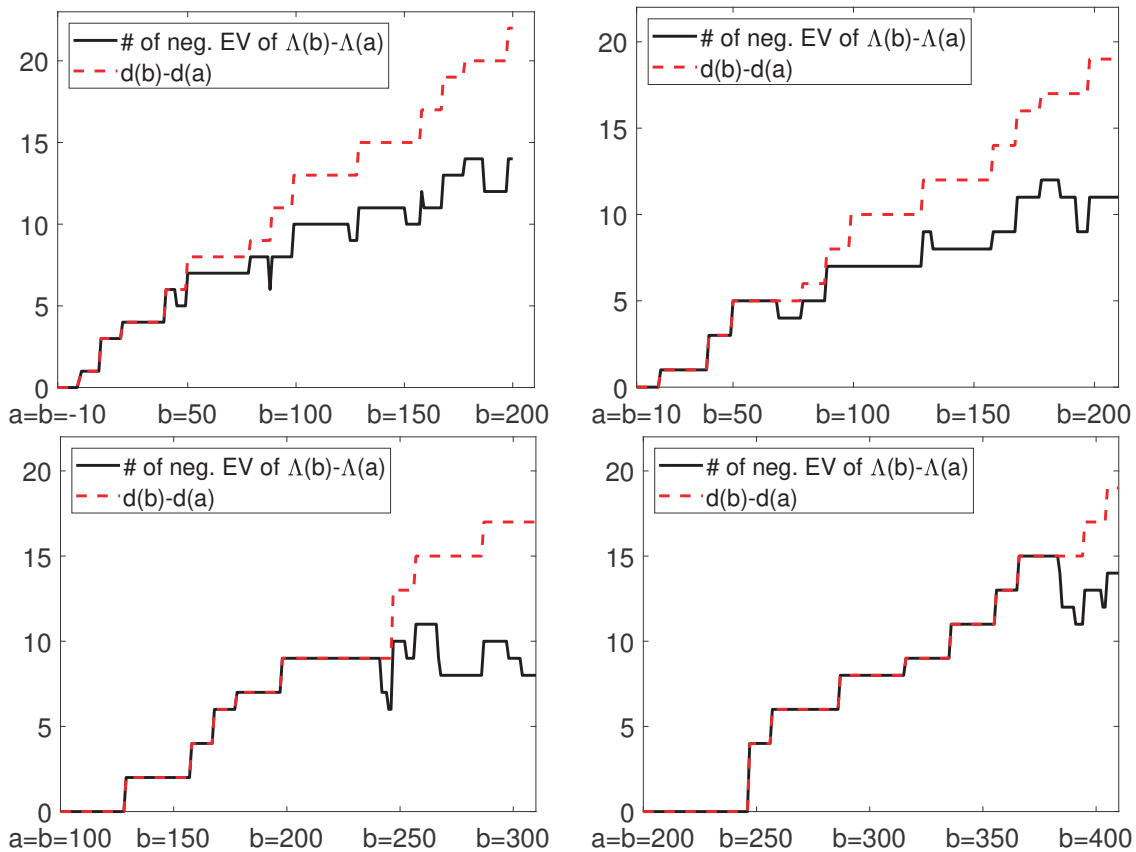

FIG. 1. Comparison of the numerically calculated number of negative eigenvalues of $\Lambda(b)-\Lambda(a)$ with the theoretical bound $d(b)-d(a)$.

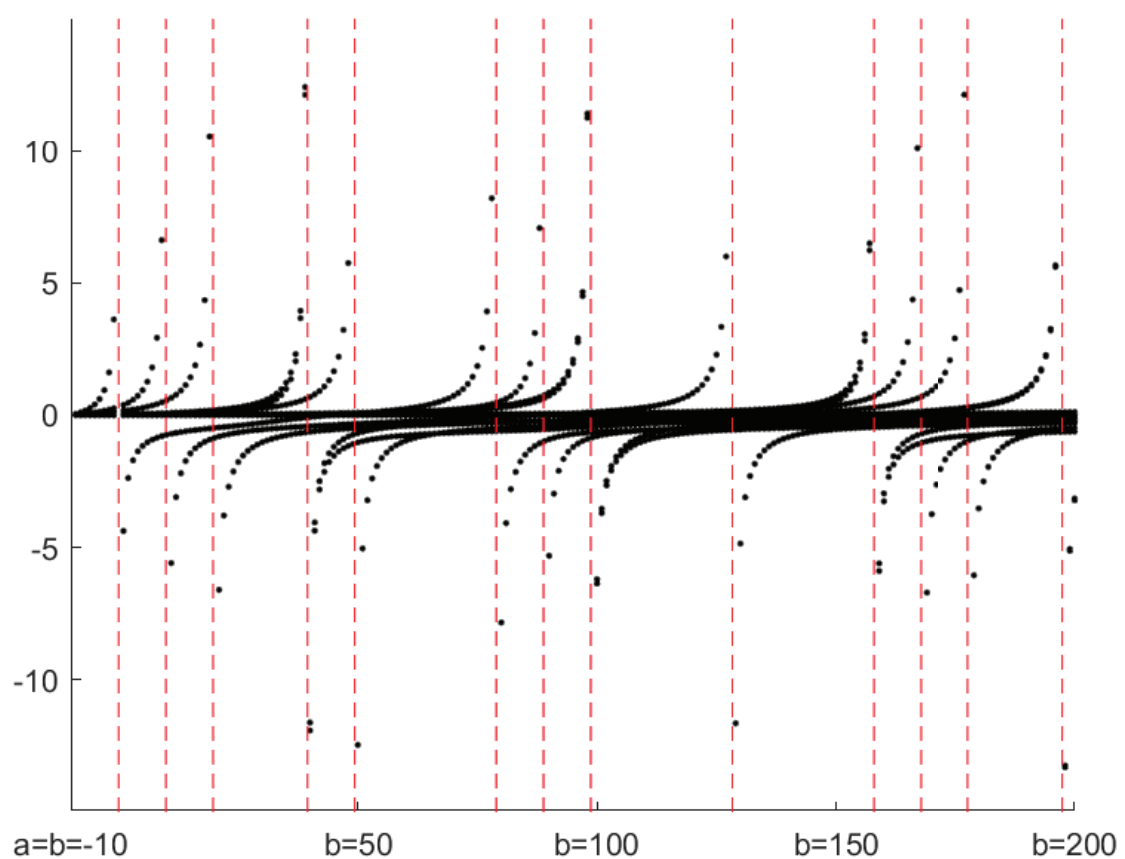

Fig. 2. Plot of the numerically calculated eigenvalues of $\Lambda(b)-\Lambda(a)$ (black dots) as a function of $b$ for fixed $a:=-10$. 
observed drop in the number of negative eigenvalues really exists. $\Lambda(b)-\Lambda(a)$ is a compact operator with an infinite number of eigenvalues accumulating at zero, and we cannot rigorously rule out the possibility that there exist more negative eigenvalues (up to the theoretically proven bound $d(b)-d(a)$ ) that we did not find due to their absolute values being below the numerical precision level.

\section{REFERENCES}

[1] L. Arnold And B. Harrach, Unique shape detection in transient eddy current problems, Inverse Problems, 29 (2013), 095004.

[2] A. Barth, B. Harrach, N. Hyvönen, and L. Mustonen, Detecting stochastic inclusions in electrical impedance tomography, Inverse Problems, 33 (2017), 115012.

[3] F. A. Berezin and M. Shubin, The Schrödinger Equation, Math. Appl. 66, Springer, New York, 2012.

[4] T. Brander, B. Harrach, M. Kar, and M. Salo, Monotonicity and enclosure methods for the p-Laplace equation, SIAM J. Appl. Math., 78 (2018), pp. 742-758, https://doi.org/10. $1137 / 17 \mathrm{M} 1128599$.

[5] N. Dunford and J. T. Schwartz, Linear Operators II, Interscience Publishers, Geneva, Switzerland, 1967.

[6] H. GARDE, Comparison of linear and non-linear monotononicity-based shape reconstruction using exact matrix characterizations, Inverse Probl. Sci. Eng., 24 (2016), pp. 524-541.

[7] H. Garde And S. Staboulis, Convergence and regularization for monotonicity-based shape reconstruction in electrical impedance tomography, Numer. Math., 135 (2017), pp. 12211251.

[8] H. Garde and S. Staboulis, The regularized monotonicity method: Detecting irregular indefinite inclusions, Inverse Probl. Imaging, 13 (2019), pp. 93-116.

[9] B. Gebauer, Localized potentials in electrical impedance tomography, Inverse Probl. Imaging, 2 (2008), pp. 251-269.

[10] F. Gesztesy and M. Mitrea, Generalized Robin boundary conditions, Robin-to-Dirichlet maps, and Krein-type resolvent formulas for Schrödinger operators on bounded Lipschitz domains, in Perspectives in Partial Differential Equations, Harmonic Analysis and Applications, Proc. Sympos. Pure Math. 79, AMS, Providence, RI, 2008, pp. 105-173, https://doi.org/10.1090/pspum/079/2500491.

[11] D. S. Grebenkov and B.-T. NGuyen, Geometrical structure of laplacian eigenfunctions, SIAM Rev., 55 (2013), pp. 601-667.

[12] R. Griesmaier and B. Harrach, Monotonicity in inverse medium scattering on unbounded domains, SIAM J. Appl. Math., 78 (2018), pp. 2533-2557.

[13] G. H. Hardy and E. M. Wright, An Introduction to the Theory of Numbers, 6th ed., Oxford University Press, Oxford, 2008.

[14] B. Harrach, On uniqueness in diffuse optical tomography, Inverse Problems, 25 (2009), 055010 .

[15] B. HaRrach, Simultaneous determination of the diffusion and absorption coefficient from boundary data, Inverse Probl. Imaging, 6 (2012), pp. 663-679.

[16] B. Harrach, Uniqueness and Lipschitz stability in electrical impedance tomography with finitely many electrodes, Inverse Problems, 35 (2019), 024005.

[17] B. Harrach, E. Lee, and M. Ullrich, Combining frequency-difference and ultrasound modulated electrical impedance tomography, Inverse Problems, 31 (2015), 095003.

[18] B. Harrach And Y.-H. Lin, Monotonicity-based inversion of the fractional Schrödinger equation I. Positive potentials, SIAM J. Math. Anal., to appear.

[19] B. HaRrach AND Y.-H. Lin, Monotonicity-Based Inversion of the Fractional Schrödinger Equation II. General Potentials and Stability, preprint, https://arxiv.org/abs/1903.08771, 2019.

[20] B. Harrach, Y.-H. Lin, And H. Liu, On localizing and concentrating electromagnetic fields, SIAM J. Appl. Math., 78 (2018), pp. 2558-2574.

[21] B. Harrach and H. Meftahi, Global uniqueness and Lipschitz-stability for the inverse Robin transmission problem, SIAM J. Appl. Math., 79 (2019), pp. 525-550.

[22] B. Harrach AND M. N. Minh, Enhancing residual-based techniques with shape reconstruction features in electrical impedance tomography, Inverse Problems, 32 (2016), 125002.

[23] B. HaRRACh AND M. N. Minh, Monotonicity-based regularization for phantom experiment data in electrical impedance tomography, in New Trends in Parameter Identification for Mathematical Models, Springer, New York, 2018, pp. 107-120. 
[24] B. Harrach, V. Pohjola, and M. Salo, Monotonicity and local uniqueness for the Helmholtz equation, Anal. PDE, to appear.

[25] B. HaRrach AND J. K. SEO, Exact shape-reconstruction by one-step linearization in electrical impedance tomography, SIAM J. Math. Anal., 42 (2010), pp. 1505-1518.

[26] B. Harrach AND M. Ullrich, Monotonicity-based shape reconstruction in electrical impedance tomography, SIAM J. Math. Anal., 45 (2013), pp. 3382-3403.

[27] B. Harrach And M. UlLRICH, Resolution guarantees in electrical impedance tomography, IEEE Trans. Med. Imaging, 34 (2015), pp. 1513-1521.

[28] B. HaRrach AND M. Ullrich, Local uniqueness for an inverse boundary value problem with partial data, Proc. Amer. Math. Soc., 145 (2017), pp. 1087-1095.

[29] T. Kato, Perturbation theory for linear operators, Classics Math., Springer, New York, 1995.

[30] A. Maffucci, A. Vento, S. Ventre, and A. Tamburrino, A novel technique for evaluating the effective permittivity of inhomogeneous interconnects based on the monotonicity property, IEEE Trans. Compon. Packag. Manuf. Tech., 6 (2016), pp. 1417-1427.

[31] M. Reed And B. Simon, Methods of Modern Mathematical Physics, I: Functional Analysis, Academic Press, New York, 1972.

[32] M. Reed And B. Simon, Methods of Modern Mathematical Physics. IV: Analysis of Operators, Academic Press, New York, 1978.

[33] R. Remmert, Theory of Complex Functions, Grad. Texts in Math 122, Springer, New York, 1991, https://doi.org/10.1007/978-1-4612-0939-3.

[34] M. Renardy and R. C. Rogers, An Introduction to Partial Differential Equations, 2nd ed., Texts Appl. Math. 13, Springer, New York, 2004.

[35] J. K. Seo, K. C. Kim, A. Jargal, K. Lee, and B. Harrach, A learning-based method for solving ill-posed nonlinear inverse problems: A simulation study of lung EIT, SIAM J. Imaging Sci., to appear.

[36] Z. Su, L. Udpa, G. Giovinco, S. Ventre, and A. Tamburrino, Monotonicity principle in pulsed eddy current testing and its application to defect sizing, in Proceedings of the International Review of Progress in Applied Computational Electromagnetics, IEEE, New York, 2017, pp. 1-2.

[37] A. Tamburrino and G. RubinacCi, A new non-iterative inversion method for electrical resistance tomography, Inverse Problems, 18 (2002), 1809.

[38] A. Tamburrino, Z. Sua, S. Ventre, L. UdPa, and S. S. UdPa, Monotonicity based imaging method in time domain eddy current testing, Electromagnetic Nondestructive Evaluation (XIX), 41 (2016).

[39] S. Ventre, A. Maffucci, F. Caire, N. Le Lostec, A. Perrotta, G. Rubinacci, B. Sartre, A. Vento, and A. Tamburrino, Design of a real-time eddy current tomography system, IEEE Trans. Magnetics, 53 (2017), pp. 1-8.

[40] L. Zhou, B. Harrach, and J. K. Seo, Monotonicity-based electrical impedance tomography for lung imaging, Inverse Problems, 34 (2018), 045005. 